\title{
AB INITIO FORCE FIELDS OF ALANINE DIPEPTIDE IN FOUR NON-HYDROGEN BONDED CONFORMATIONS
}

\author{
T.C. CHEAM and S. KRIMM
}

Biophysics Research Division and Department of Physics, University of Michigan, Ann Arbor, MI 48109 (U.S.A.)

(Received 30 June 1989)

\begin{abstract}
We have previously calculated the force fields and dipole-moment derivatives of the L-alanyl dipeptide, $\mathrm{CH}_{3} \mathrm{CONHCHCH}_{3} \mathrm{CONHCH}_{3}$, in the $\mathrm{C}_{5}, \mathrm{C}_{7}^{\mathrm{eq}}$, and $\mathrm{C}_{7}^{\mathrm{ax}}$ conformations with intramolecular $\mathrm{NH} \cdots \mathrm{OC}$ hydrogen bonding. We have now extended these studies with similar ones on four open, non-hydrogen-bonded conformers optimized by Scarsdale et al. - the $\beta_{2}, \alpha_{\mathrm{R}}, \alpha_{\mathrm{L}}$ and $\alpha^{\prime}$ structures. The force constants were derived using the 4-21 Gaussian basis set and were scaled as before. The present results help to isolate the effects of conformation on the force fields and dipole derivatives, and also provide further insight into the effects of hydrogen bonding by comparison with the previous results. Additional effects can also be seen, such as those of the non-planarity of the peptide group, and the influence on the $\mathrm{CO}$ bond when the adjacent $\mathrm{NH}$ forms a hydrogen bond, and vice versa.
\end{abstract}

\section{INTRODUCTION}

In previous papers we have given the results of ab initio calculations of the force fields and dipole-moment derivatives of the glycine [1] and alanine [2] dipeptides, $\mathrm{CH}_{3} \mathrm{CONHCHRCONHCH}\left(\mathrm{R}=\mathrm{H}, \mathrm{CH}_{3}\right)$, in the $\mathrm{C}_{5}$ and $\mathrm{C}_{7}$ conformations with intramolecular hydrogen bonding. These optimized conformations were obtained by Schäfer and co-workers [3,4] using the 4-21 Gaussian basis set. These workers also derived several conformers of Gly and Ala dipeptides in which $\mathrm{NH} \cdots$ OC hydrogen bonds are not present [3-6]. To see how the force fields vary with conformation alone, without additional complications from changes in hydrogen bonding, we performed calculations on four of these "open" conformers of Ala dipeptide denoted by Scarsdale et al. [4] as the $\beta_{2}, \alpha_{\mathrm{R}}, \alpha_{\mathrm{L}}$, and $\alpha^{\prime}$ structures. Besides helping to isolate the effects of conformation on the force constants and normal modes, one can also gain further insight into the effects of hydrogen bonding by comparison with earlier results on the $\mathrm{C}_{5}, \mathrm{C}_{7}^{\mathrm{eq}}$, and $\mathrm{C}_{7}^{\mathrm{ax}}$ Ala dipeptide conformers. It is hoped that these forcefield calculations on nine conformations of Gly and Ala dipeptides, together with the geometry optimization studies of Schäfer and co-workers, will provide 
a large set of data for help in developing more reliable molecular mechanics energy functions for peptides and polypeptides [7].

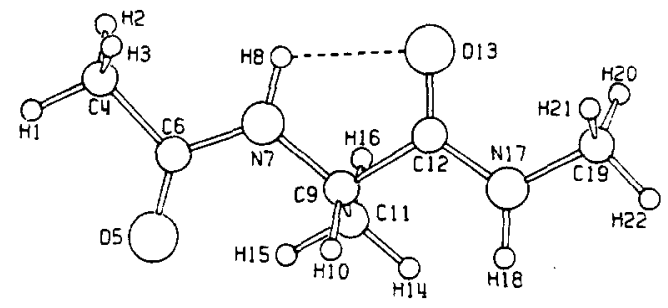

C5 $(-166,167)$

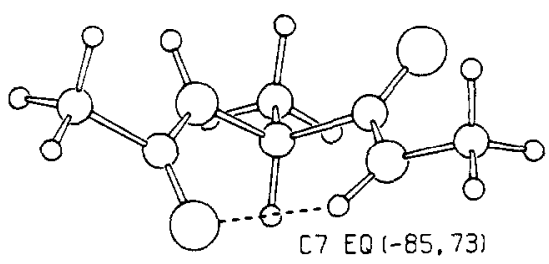

C7 EQ $(-85.73)$
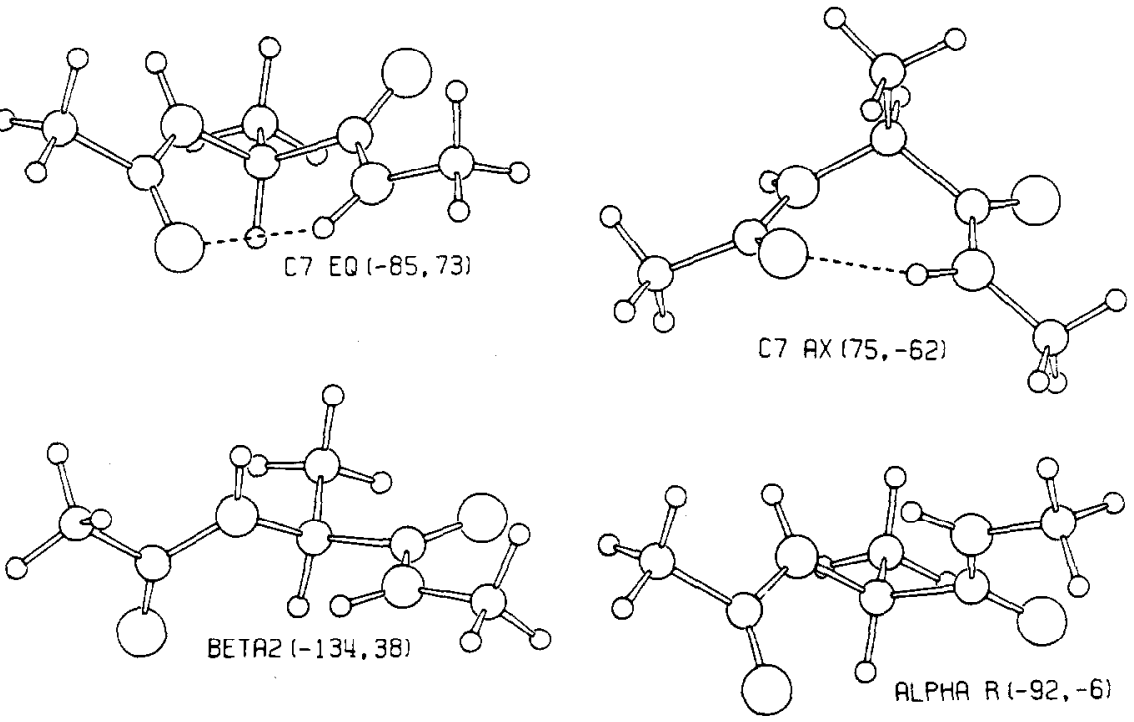

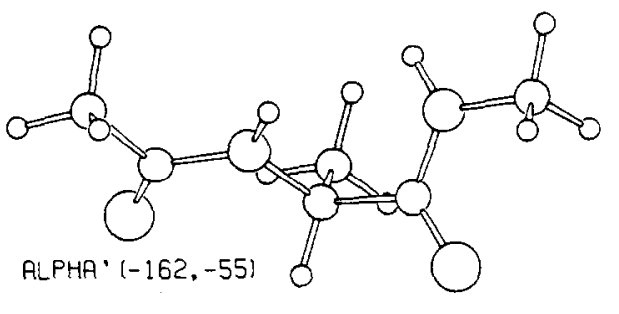

Fig. 1. Seven optimized conformations of Ala dipeptide, with the $\phi$ and $\psi$ angles (in degrees) indicated. 
TABLE 1

Internal coordinates and molecular dimensions

\begin{tabular}{|c|c|c|c|c|c|}
\hline $\mathrm{R}$ & Atoms & $\beta_{2}$ & $\alpha_{\mathrm{R}}$ & $\alpha_{\mathbf{L}}$ & $\alpha^{\prime}$ \\
\hline \multicolumn{6}{|c|}{ Bond stretches $(\AA)$} \\
\hline 1 & $4-6$ & 1.518 & 1.518 & 1.518 & 1.518 \\
\hline 2 & $6-7$ & 1.369 & 1.371 & 1.369 & 1.362 \\
\hline 3 & $7-9$ & 1.467 & 1.463 & 1.470 & 1.471 \\
\hline 4 & $6-5$ & 1.220 & 1.218 & 1.219 & 1.222 \\
\hline 5 & $7-8$ & 0.996 & 0.995 & 0.994 & 0.994 \\
\hline 6 & $9-12$ & 1.534 & 1.534 & 1.538 & 1.526 \\
\hline 7 & $12-17$ & 1.349 & 1.344 & 1.351 & 1.360 \\
\hline 8 & $17-19$ & 1.463 & 1.463 & 1.463 & 1.466 \\
\hline 9 & $12-13$ & 1.223 & 1.226 & 1.220 & 1.220 \\
\hline 10 & $17-18$ & 0.992 & 0.991 & 0.991 & 0.993 \\
\hline 11 & $4-1$ & 1.077 & 1.077 & 1.077 & 1.077 \\
\hline 12 & $4-2$ & 1.083 & 1.083 & 1.083 & 1.083 \\
\hline 13 & $4-3$ & 1.083 & 1.083 & 1.083 & 1.083 \\
\hline 14 & $9-11$ & 1.532 & 1.539 & 1.533 & 1.541 \\
\hline 15 & $9-10$ & 1.081 & 1.079 & 1.083 & 1.077 \\
\hline 16 & $11-14$ & 1.079 & 1.079 & 1.079 & 1.082 \\
\hline 17 & $11-15$ & 1.081 & 1.082 & 1.082 & 1.079 \\
\hline 18 & $11-16$ & 1.083 & 1.084 & 1.078 & 1.082 \\
\hline 19 & $19-20$ & 1.080 & 1.082 & 1.080 & 1.080 \\
\hline 20 & $19-21$ & 1.082 & 1.079 & 1.081 & 1.081 \\
\hline 21 & $19-22$ & 1.080 & 1.080 & 1.080 & 1.080 \\
\hline \multicolumn{6}{|c|}{ Angle bends $\left({ }^{\circ}\right)$} \\
\hline 22 & $4-6-7$ & 114.4 & 114.6 & 114.7 & 114.8 \\
\hline 23 & $4-6-5$ & 123.2 & 123.4 & 123.3 & 123.3 \\
\hline 24 & $7-6-5$ & 122.4 & 122.1 & 122.1 & 121.9 \\
\hline 25 & $6-7-9$ & 121.4 & 121.2 & 121.4 & 120.4 \\
\hline 26 & $6-7-8$ & 119.3 & 119.3 & 119.1 & 120.3 \\
\hline 27 & $9-7-8$ & 117.8 & 118.1 & 118.1 & 118.8 \\
\hline 28 & $9-12-17$ & 115.5 & 117.2 & 115.2 & 115.2 \\
\hline 29 & $9-12-13$ & 121.7 & 119.4 & 121.6 & 122.7 \\
\hline 30 & $17-12-13$ & 122.7 & 123.4 & 123.0 & 122.2 \\
\hline 31 & $12-17-19$ & 120.3 & 120.3 & 120.1 & 119.8 \\
\hline 32 & $12-17-18$ & 119.4 & 119.1 & 119.5 & 120.3 \\
\hline 33 & $19-17-18$ & 120.3 & 120.2 & 119.9 & 119.2 \\
\hline 34 & $6-4-1$ & 108.6 & 108.6 & 108.5 & 108.6 \\
\hline 35 & $6-4-2$ & 110.0 & 110.2 & 110.4 & 110.2 \\
\hline 36 & $6-4-3$ & 110.6 & 110.6 & 110.4 & 110.4 \\
\hline 37 & $1-4-2$ & 109.4 & 109.4 & 109.6 & 109.5 \\
\hline 38 & $1-4-3$ & 109.7 & 109.6 & 109.5 & 109.6 \\
\hline 39 & $2-4-3$ & 108.5 & 108.4 & 108.5 & 108.6 \\
\hline 40 & $7-9-12$ & 110.6 & 114.0 & 111.1 & 108.5 \\
\hline 41 & $7-9-10$ & 105.4 & 108.0 & 105.8 & 108.8 \\
\hline 42 & $12-9-10$ & 107.8 & 106.0 & 106.0 & 106.7 \\
\hline 43 & $11-9-10$ & 109.9 & 109.8 & 109.7 & 109.4 \\
\hline
\end{tabular}


TABLE 1 (continued)

\begin{tabular}{|c|c|c|c|c|c|}
\hline $\mathbf{R}$ & Atoms & $\beta_{2}$ & $\alpha_{\mathrm{R}}$ & $\alpha_{\mathrm{L}}$ & $\alpha^{\prime}$ \\
\hline 44 & $7-9-11$ & 112.2 & 110.6 & 113.2 & 111.7 \\
\hline 45 & $12-9-11$ & 110.7 & 108.4 & 110.6 & 111.6 \\
\hline 46 & $9-11-14$ & 109.9 & 108.8 & 109.6 & 110.2 \\
\hline 47 & $9-11-15$ & 109.6 & 110.3 & 109.8 & 108.5 \\
\hline 48 & $9-11-16$ & 110.5 & 110.6 & 109.5 & 111.1 \\
\hline 49 & $14-11-15$ & 109.6 & 109.5 & 109.5 & 109.1 \\
\hline 50 & $14-11-16$ & 108.0 & 108.5 & 108.6 & 109.0 \\
\hline 51 & $15-11-16$ & 109.1 & 109.1 & 109.7 & 109.0 \\
\hline 52 & $17-19-20$ & 110.2 & 111.2 & 110.0 & 110.1 \\
\hline 53 & $17-19-21$ & 110.9 & 109.4 & 110.9 & 110.8 \\
\hline 54 & $17-19-22$ & 108.8 & 108.9 & 108.8 & 108.8 \\
\hline 55 & $20-19-21$ & 108.3 & 108.4 & 108.4 & 108.3 \\
\hline 56 & $20-19-22$ & 109.4 & 109.3 & 109.4 & 109.4 \\
\hline 57 & $21-19-22$ & 109.3 & 109.6 & 109.4 & 109.5 \\
\hline \multicolumn{6}{|c|}{ Out-of-plane bends $\left(^{\circ}\right)$} \\
\hline 58 & $6-5$ & -0.4 & -0.1 & 1.0 & 0.3 \\
\hline 59 & $7-8$ & -12.6 & -12.2 & 11.9 & 7.5 \\
\hline 60 & $12-13$ & -1.9 & 1.9 & -4.6 & 0.1 \\
\hline 61 & $17-18$ & -0.4 & 6.4 & -7.5 & -8.8 \\
\hline \multicolumn{6}{|c|}{ Torsions } \\
\hline 62 & $4-6$ & & & & \\
\hline 63 & $6-7$ & & & & \\
\hline 64 & $7-9$ & & & & \\
\hline 65 & $9-12$ & & & & \\
\hline 66 & $12-17$ & & & & \\
\hline 67 & $17-19$ & & & & \\
\hline 68 & $9-11$ & & & & \\
\hline
\end{tabular}

aPositive: atoms C6, N17 move out of the $C_{5}$ plane in Fig. 1: N7, C12 move below the plane.

\section{METHOD OF CALCULATION}

The four open conformers of Ala dipeptide used in the calculations are shown in Fig. 1, where their $\phi$ (C6-N7-C9-C12) and $\psi(\mathrm{N} 7-\mathrm{C} 9-\mathrm{C} 12-\mathrm{N} 17)$ dihedral angles are indicated. Also shown, for ease of comparison, are the $\mathrm{C}_{5}, \mathrm{C}_{7}^{\text {eq }}$, and $\mathrm{C}_{7}^{\mathrm{ax}}$ conformers studied previously. The 4-21 SCF energies of the $\beta_{2}, \alpha_{\mathrm{R}}, \alpha_{\mathrm{L}}$, and $\alpha^{\prime}$ structures are, respectively, 3.9, 4.9,6.7, and $7.9 \mathrm{kcal} \mathrm{mol}^{-1}$ above that of the $\mathrm{C}_{7}^{\mathrm{eq}}$; the corresponding figures for $\mathrm{C}_{5}$ and $\mathrm{C}_{7}^{\mathrm{ax}}$ are 1.4 and $2.6 \mathrm{kcal} \mathrm{mol}^{-1}$ [4]. It may be noted that the $\phi$ or $\psi$ angles are similar in several pairs of conformers: $\phi$ in $\mathrm{C}_{5}$ and $\alpha^{\prime}, \phi$ in $\mathrm{C}_{7}^{\mathrm{eq}}$ and $\alpha_{\mathrm{R}}, \phi$ in $\mathrm{C}_{7}^{\mathrm{ax}}$ and $\alpha_{\mathrm{L}}, \psi$ in $\mathrm{C}_{7}^{\mathrm{ax}}$ and $\alpha^{\prime}$, and $\psi$ in $\beta_{2}$ and $\alpha_{\mathrm{L}}$. These relations can be useful in looking for regularities in the variations with $\phi, \psi$ or hydrogen bonding in the geometries, force constants and normal modes. 
The primitive internal coordinates and equilibrium molecular dimensions are listed in Table 1. The quadratic force constants and dipole-moment derivatives were computed with the 4-21 basis set [8] as in Ref. 1, using two-sided displacements along group, or local symmetry, coordinates. These group coordinates for Ala dipeptide, constructed from the primitive internal coordinates, are as given in Ref. 2. Because of the sizeable deviations from planarity in several of the out-of-plane bends (Table 1), we have used the expressions for the B matrix elements appropriate for non-planar groups [9]. The force constants were scaled as in our previous papers, using the set of six scale factors derived for some amides by Fogarasi and Balázs [10], two of which we modified [1].

\section{RESULTS AND DISCUSSION}

The scaled diagonal and off-diagonal force constants in the group-coordinate basis are given in Tables 2 and 3 for all seven Ala dipeptide conformations; only off-diagonal terms of magnitude $\geqslant 0.05$ in at least one of the conformations are shown. The harmonic frequencies, infrared intensities, and potentialenergy distributions for the $\beta_{2}, \alpha_{\mathrm{R}}, \alpha_{\mathrm{L}}$ and $\alpha^{\prime}$ structures computed with all $\left|F_{i j}\right| \geqslant 0.01$ are listed in Table 4 .

The NH stretch2 (str2) force constant is very nearly the same in $\mathrm{C}_{5}$ and all the open conformations, where $\mathrm{N} 17 \mathrm{H} 18$ is free. This shows that the significant decreases in this term in $\mathrm{C}_{7}^{\mathrm{eq}}$ and $\mathrm{C}_{7}^{\mathrm{ax}}$ are due entirely to hydrogen bonding. The free $\mathrm{NH}$ str 1 term in the $\mathrm{C}_{7}$ and open conformations varies slightly; among the latter its value is lowest in $\alpha_{\mathrm{R}}$, where $\mathrm{N} 7 \mathrm{H} 8$ is most nearly cis to $\mathrm{C}^{\alpha} \mathrm{C}^{\beta}$ (Fig. 1 ), indicating the effect of the side group [11-13]. The difference in $\mathrm{f}(\mathrm{NH}$ str1) between $\mathrm{C}_{7}^{\mathrm{ax}}$ and $\alpha_{\mathrm{L}}$, even though the $\mathrm{NH}$ and side chain are oriented similarly in both structures, may be a result of the hydrogen bonding; the somewhat higher $\mathrm{f}(\mathrm{NH} \operatorname{str} 1)$ in $\mathrm{C}_{7}^{\text {eq }}$ compared with $\alpha_{\mathrm{R}}$ is consistent with this relation, particularly when we recall that the hydrogen bond is strongest in $\mathrm{C}_{7}^{\text {ax }}$ [2]. Another likely reason for these differences between $\mathrm{C}_{7}$ and $\alpha$ may be the higher degree of non-planarity at N7 in $\alpha_{\mathrm{R}}$ and $\alpha_{\mathrm{L}}$ (Table 1). That the free $\mathrm{f}(\mathrm{NH}$ str1) is systematically lower than the free $\mathrm{f}(\mathrm{NH}$ str2) may be due to the different substituents. This difference in the free $\mathrm{NH}$ stretch terms means that even open conformations of Ala dipeptide can exhibit two NH str frequencies, whose separation can be as much as $64 \mathrm{~cm}^{-1}$ (Table 4 ), thereby possibly complicating the identification of hydrogen-bonded structures in solution [11-13 and references therein]. However, our results indicate that although the $\mathrm{C}_{5}$ conformer may indeed be difficult to distinguish from open conformers using the $\mathrm{NH}$ stretch bands, the $\mathrm{C}_{7}$ conformers have bonded $\mathrm{NH}$ stretch frequencies well below $3400 \mathrm{~cm}^{-1}$ [2], whereas the free $\mathrm{NH}$ stretch frequencies in all conformations are above $3400 \mathrm{~cm}^{-1}$. Moreover, the infrared intensities (Table 4) of both NH stretch modes in the open conformers are expected to be much 
TABLE 2

Scaled diagonal force constants of Ala dipeptides ${ }^{\mathrm{a}}$

\begin{tabular}{|c|c|c|c|c|c|c|c|}
\hline & $\mathrm{C}_{5}$ & $\mathrm{C}_{7}^{\mathrm{eq}}$ & $\mathrm{C}_{7}^{\operatorname{ax}}$ & $\beta_{2}$ & $\alpha_{\mathrm{R}}$ & $\alpha_{\mathrm{L}}$ & $\alpha^{\prime}$ \\
\hline NH str1 & 6.363 & 6.453 & 6.577 & 6.521 & 6.415 & 6.448 & 6.471 \\
\hline CO str 1 & 11.040 & 10.652 & 10.550 & 11.400 & 11.542 & 11.486 & 11.300 \\
\hline CN str1 & 6.661 & 6.635 & 6.631 & 6.186 & 6.121 & 6.108 & 6.382 \\
\hline MC str & 4.170 & 4.187 & 4.167 & 4.169 & 4.169 & 4.165 & 4.159 \\
\hline $\mathrm{NC}^{\alpha}$ str & 5.168 & 4.856 & 4.922 & 4.799 & 4.857 & 4.832 & 4.847 \\
\hline NH str2 & 6.666 & 6.239 & 6.134 & 6.607 & 6.656 & 6.632 & 6.676 \\
\hline CO str2 & 10.922 & 11.026 & 10.965 & 11.221 & 11.044 & 11.385 & 11.479 \\
\hline CN str2 & 6.794 & 6.901 & 7.032 & 6.692 & 6.792 & 6.573 & 6.405 \\
\hline $\mathrm{C}^{\alpha} \mathrm{C}$ str & 4.259 & 4.126 & 4.039 & 4.166 & 4.078 & 4.135 & 4.139 \\
\hline NM str & 5.206 & 5.289 & 5.282 & 5.235 & 5.260 & 5.272 & 5.218 \\
\hline MH1 str & 4.971 & 4.982 & 4.991 & 4.981 & 4.984 & 4.980 & 4.982 \\
\hline MH2 str & 4.796 & 4.804 & 4.785 & 4.781 & 4.778 & 4.787 & 4.781 \\
\hline MH3 str & 4.797 & 4.789 & 4.805 & 4.794 & 4.791 & 4.779 & 4.790 \\
\hline $\mathrm{C}^{\alpha} \mathrm{C}^{\beta}$ str & 4.088 & 4.373 & 4.131 & 4.283 & 4.246 & 4.289 & 4.105 \\
\hline $\mathrm{C}^{\alpha} \mathrm{H}^{\alpha}$ str & 4.801 & 4.918 & 4.941 & 4.904 & 4.926 & 4.755 & 4.965 \\
\hline $\mathrm{CH} 16$ str & 4.822 & 4.872 & 5.042 & 4.780 & 4.731 & 4.992 & 4.779 \\
\hline CH14 str & 4.778 & 4.865 & 4.812 & 4.940 & 4.945 & 4.932 & 4.799 \\
\hline $\mathrm{CH} 15 \mathrm{str}$ & 4.946 & 4.804 & 4.782 & 4.814 & 4.801 & 4.772 & 4.938 \\
\hline MH22 str & 4.875 & 4.857 & 4.860 & 4.848 & 4.830 & 4.831 & 4.856 \\
\hline MH20 str & 4.820 & 4.799 & 4.756 & 4.821 & 4.744 & 4.822 & 4.833 \\
\hline MH21 str & 4.820 & 4.751 & 4.796 & 4.768 & 4.875 & 4.782 & 4.797 \\
\hline MCN def & 0.946 & 0.968 & 1.034 & 0.993 & 0.987 & 0.974 & 0.979 \\
\hline $\mathrm{CO} \mathrm{ibl}$ & 1.153 & 1.208 & 1.242 & 1.183 & 1.146 & 1.143 & 1.163 \\
\hline $\mathrm{CNC}^{\alpha}$ def & 0.924 & 1.149 & 1.432 & 0.952 & 0.910 & 1.001 & 0.952 \\
\hline NH ib1 & 0.553 & 0.585 & 0.614 & 0.597 & 0.595 & 0.601 & 0.586 \\
\hline $\mathrm{C}^{\alpha} \mathrm{CN}$ def & 1.018 & 1.164 & 1.256 & 1.117 & 1.111 & 1.088 & 1.047 \\
\hline $\mathrm{CO}$ ib2 & 1.276 & 1.163 & 1.107 & 1.187 & 1.115 & 1.169 & 1.110 \\
\hline CNM def & 0.794 & 0.814 & 0.826 & 0.799 & 0.814 & 0.805 & 0.814 \\
\hline $\mathrm{NH}$ ib2 & 0.585 & 0.656 & 0.659 & 0.573 & 0.564 & 0.575 & 0.583 \\
\hline M1 sb & 0.549 & 0.551 & 0.551 & 0.550 & 0.549 & 0.549 & 0.549 \\
\hline M1 ab1 & 0.521 & 0.519 & 0.519 & 0.521 & 0.521 & 0.520 & 0.521 \\
\hline $\mathrm{M} 1 \mathrm{ab} 2$ & 0.522 & 0.521 & 0.522 & 0.523 & 0.522 & 0.523 & 0.523 \\
\hline M1 rock1 & 0.649 & 0.652 & 0.656 & 0.651 & 0.649 & 0.651 & 0.649 \\
\hline M1 rock2 & 0.605 & 0.607 & 0.606 & 0.607 & 0.606 & 0.602 & 0.604 \\
\hline $\mathrm{NC}^{\alpha} \mathrm{C}$ def & 1.020 & 1.043 & 1.882 & 0.991 & 1.029 & 1.152 & 1.124 \\
\hline $\mathrm{H}^{\alpha} \mathrm{bl}$ & 0.637 & 0.666 & 0.708 & 0.649 & 0.641 & 0.651 & 0.657 \\
\hline $\mathrm{H}^{\alpha} \mathrm{b} 2$ & 0.697 & 0.736 & 0.744 & 0.691 & 0.703 & 0.723 & 0.708 \\
\hline $\mathrm{C}^{\beta} \mathrm{b} 1$ & 1.393 & 1.410 & 1.116 & 1.325 & 1.369 & 1.342 & 1.036 \\
\hline $\mathrm{C}^{\beta} \mathrm{b} 2$ & 1.109 & 1.134 & 0.956 & 1.127 & 1.065 & 1.129 & 1.012 \\
\hline $\mathrm{C}^{\beta} \mathrm{sb}$ & 0.556 & 0.556 & 0.560 & 0.558 & 0.555 & 0.561 & 0.556 \\
\hline $\mathrm{C}^{\beta} \mathrm{ab1}$ & 0.539 & 0.533 & 0.533 & 0.534 & 0.539 & 0.526 & 0.544 \\
\hline $\mathrm{C}^{\beta} \mathrm{ab2}$ & 0.536 & 0.533 & 0.545 & 0.531 & 0.542 & 0.538 & 0.534 \\
\hline $\mathrm{C}^{\beta}$ rock1 & 0.669 & 0.673 & 0.690 & 0.671 & 0.674 & 0.688 & 0.669 \\
\hline $\mathrm{C}^{\beta}$ rock2 & 0.649 & 0.664 & 0.674 & 0.671 & 0.655 & 0.672 & 0.648 \\
\hline
\end{tabular}


TABLE 2 (continued)

\begin{tabular}{|c|c|c|c|c|c|c|c|}
\hline & $\mathrm{C}_{5}$ & $\mathrm{C}_{7}^{\mathrm{eq}}$ & $\mathrm{C}_{7}^{\mathrm{ax}}$ & $\beta_{2}$ & $\alpha_{R}$ & $\alpha_{\mathrm{L}}$ & $\alpha^{\prime}$ \\
\hline M2 sb & 0.607 & 0.602 & 0.601 & 0.604 & 0.602 & 0.603 & 0.606 \\
\hline M2 abl & 0.544 & 0.548 & 0.548 & 0.547 & 0.546 & 0.547 & 0.545 \\
\hline M2 ab2 & 0.535 & 0.540 & 0.541 & 0.538 & 0.538 & 0.538 & 0.537 \\
\hline M2 rock1 & 0.783 & 0.781 & 0.780 & 0.782 & 0.781 & 0.781 & 0.782 \\
\hline M2 rock2 & 0.796 & 0.792 & 0.791 & 0.796 & 0.780 & 0.795 & 0.799 \\
\hline CO ob1 & 0.777 & 0.803 & 0.825 & 0.763 & 0.761 & 0.775 & 0.778 \\
\hline NH obl & 0.220 & 0.243 & 0.293 & 0.197 & 0.208 & 0.237 & 0.206 \\
\hline $\mathrm{CO}$ ob2 & 0.802 & 0.790 & 0.787 & 0.786 & 0.779 & 0.806 & 0.816 \\
\hline $\mathrm{NH}$ ob2 & 0.234 & 0.255 & 0.303 & 0.233 & 0.205 & 0.194 & 0.206 \\
\hline $\mathrm{MC}$ tor & 0.060 & 0.058 & 0.062 & 0.064 & 0.065 & 0.065 & 0.063 \\
\hline $\mathrm{CN}$ tor 1 & 0.446 & 0.546 & 0.561 & 0.404 & 0.419 & 0.462 & 0.435 \\
\hline $\mathrm{NC}^{\alpha}$ tor & 0.385 & 0.544 & 0.724 & 0.272 & 0.312 & 0.451 & 0.359 \\
\hline $\mathrm{C}^{\alpha} \mathrm{C}$ tor & 0.320 & 0.397 & 0.552 & 0.294 & 0.344 & 0.422 & 0.357 \\
\hline $\mathrm{CN}$ tor 2 & 0.374 & 0.498 & 0.522 & 0.417 & 0.416 & 0.388 & 0.404 \\
\hline NM tor & 0.050 & 0.054 & 0.055 & 0.051 & 0.051 & 0.053 & 0.054 \\
\hline $\mathrm{C}^{\alpha} \mathrm{C}^{\beta}$ tor & 0.163 & 0.121 & 0.142 & 0.127 & 0.157 & 0.135 & 0.153 \\
\hline
\end{tabular}

anits: stretches, mdyn $\AA^{-1}$; bends and torsions, mdyn $\AA \operatorname{rad}^{-2}$. Group coordinates are defined in Ref. 2.

weaker than those of the bonded $\mathrm{NH}$ stretch modes in the $\mathrm{C}_{5}$ and $\mathrm{C}_{7}$ structures [2]. To conclude our discussion of the $\mathrm{NH}$ stretch force constants, we note that our results do not show clear evidence of an $\mathrm{NH} \cdots \mathrm{N}$ interaction that Scarsdale et al. [4] proposed to explain certain structural relations; the H18 $\cdots \mathrm{N} 7$ distances are no shorter than $2.30 \AA\left(\alpha_{\mathrm{R}}\right)$, which would suggest a very weak interaction at best.

The CO str1 force constants in the open structures are relatively constant. The much reduced $\mathrm{f}(\mathrm{CO}$ str 1$)$ values in $\mathrm{C}_{7}^{\mathrm{eq}}$ and $\mathrm{C}_{7}^{\mathrm{ax}}$ are evidently due to the hydrogen bonding. That this term in $\mathrm{C}_{5}$ is also significantly lowered (compare especially with $\alpha^{\prime}$ where $\phi$ is nearly identical) suggests that the CO stretch force constant is affected when the adjacent $\mathrm{NH}$ forms a hydrogen bond, undoubtedly because of the electronic resonance in the peptide group. Also, the C6O5 bond is longer in $\mathrm{C}_{5}$ (and the $\mathrm{C} 6 \mathrm{~N} 7$ bond shorter) than in the open structures, and an analysis of the Mulliken populations shows an increase in the partial charge on $\mathrm{O} 5$ in the $\mathrm{C}_{5}$ conformer as compared with the open conformers. Furthermore, $\mathrm{f}\left(\mathrm{CO}\right.$ str2) is considerably lower in $\mathrm{C}_{7}^{\mathrm{ax}}$ than in $\alpha^{\prime}$, where $\psi$ is similar. These results point to the significance of cooperative electronic effects [14] in structures, in particular crystals, where both the $\mathrm{CO}$ and $\mathrm{NH}$ groups of a peptide unit participate in hydrogen bonds.

$\mathrm{CO}$ str2, being adjacent to $\mathrm{C}^{\alpha}$, exhibits more variability in the open structures; there is a distinct tendency for $\mathrm{f}(\mathrm{CO}$ str2 $)$ to increase as $\psi$ increases in magnitude (Fig. 2(a)). The $\psi$ angles are larger in $\mathrm{C}_{5}$ and $\mathrm{C}_{7}$, but the force constant may be lowered by the hydrogen bonding, either directly on $\mathrm{CO}$ str2 
TABLE 3

Scaled off-diagonal group coordinate force constants of Ala dipeptides, $\left|F_{i j}\right| \geqslant 0.05^{\mathrm{a}}$

\begin{tabular}{|c|c|c|c|c|c|c|c|}
\hline Term & $\mathrm{C}_{5}$ & $\mathrm{C}_{7}^{\mathrm{eq}}$ & $\mathrm{C}_{7}^{\mathrm{ax}}$ & $\beta_{2}$ & $\alpha_{\mathrm{R}}$ & $\alpha_{\mathrm{L}}$ & $\alpha^{\prime}$ \\
\hline $1-3$ & -0.04 & -0.05 & -0.01 & -0.05 & -0.06 & -0.06 & -0.05 \\
\hline $1-5$ & 0.13 & 0.04 & 0.0 & 0.02 & 0.02 & -0.01 & 0.01 \\
\hline 1. 7 & -0.10 & -0.01 & 0.0 & 0.0 & 0.03 & 0.05 & 0.02 \\
\hline $1-8$ & 0.05 & 0.0 & 0.0 & 0.0 & -0.01 & -0.02 & -0.04 \\
\hline $1-9$ & -0.06 & 0.0 & -0.02 & 0.0 & 0.0 & -0.02 & 0.0 \\
\hline $1-22$ & 0.0 & -0.01 & -0.05 & -0.04 & -0.03 & -0.03 & -0.03 \\
\hline $1-24$ & -0.11 & -0.09 & -0.08 & -0.10 & -0.10 & -0.09 & -0.09 \\
\hline $1-25$ & -0.11 & 0.02 & 0.04 & 0.01 & 0.02 & 0.03 & 0.02 \\
\hline $2-3$ & 1.37 & 1.36 & 1.30 & 1.34 & 1.31 & 1.28 & 1.33 \\
\hline $2-4$ & 0.36 & 0.36 & 0.38 & 0.37 & 0.37 & 0.38 & 0.37 \\
\hline $2-5$ & -0.09 & -0.09 & -0.09 & -0.11 & -0.10 & -0.10 & -0.10 \\
\hline $2-6$ & 0.0 & -0.14 & -0.17 & 0.0 & 0.01 & 0.02 & 0.0 \\
\hline $2-7$ & 0.05 & 0.05 & 0.04 & 0.07 & 0.05 & 0.03 & 0.05 \\
\hline $2-8$ & 0.0 & 0.05 & 0.06 & -0.03 & -0.03 & -0.03 & -0.02 \\
\hline $2-22$ & -0.55 & -0.55 & -0.49 & -0.52 & -0.52 & -0.51 & -0.53 \\
\hline $2-23$ & -0.16 & -0.14 & -0.05 & -0.10 & -0.11 & -0.09 & -0.13 \\
\hline $2-26$ & -0.02 & 0.08 & 0.07 & 0.0 & -0.03 & -0.03 & 0.0 \\
\hline $2-27$ & 0.05 & 0.05 & 0.06 & 0.0 & 0.02 & 0.02 & 0.0 \\
\hline $2-35$ & 0.03 & -0.02 & 0.08 & 0.02 & 0.0 & 0.03 & 0.0 \\
\hline $2-38$ & 0.04 & 0.07 & 0.03 & 0.0 & -0.02 & -0.05 & 0.0 \\
\hline $2-39$ & 0.05 & 0.0 & 0.03 & 0.03 & 0.02 & 0.0 & 0.04 \\
\hline $2-57$ & -0.02 & 0.04 & -0.02 & -0.05 & -0.05 & 0.03 & 0.0 \\
\hline $3-4$ & 0.31 & 0.30 & 0.31 & 0.30 & 0.31 & 0.31 & 0.31 \\
\hline $3-5$ & 0.20 & 0.24 & 0.30 & 0.21 & 0.18 & 0.21 & 0.20 \\
\hline $3-7$ & -0.01 & -0.02 & -0.02 & -0.04 & -0.06 & -0.05 & -0.04 \\
\hline $3-8$ & -0.03 & -0.09 & -0.11 & 0.04 & 0.05 & 0.04 & 0.02 \\
\hline $3-9$ & -0.03 & 0.04 & 0.07 & -0.01 & 0.0 & 0.0 & 0.0 \\
\hline $3-15$ & -0.04 & -0.05 & -0.02 & -0.07 & -0.06 & -0.01 & -0.03 \\
\hline $3-22$ & 0.19 & 0.16 & 0.17 & 0.15 & 0.18 & 0.19 & 0.17 \\
\hline $3-23$ & -0.45 & -0.50 & -0.49 & -0.51 & -0.47 & -0.46 & -0.47 \\
\hline $3-24$ & 0.21 & 0.22 & 0.23 & 0.24 & 0.20 & 0.19 & 0.22 \\
\hline $3-25$ & 0.17 & 0.19 & 0.22 & 0.19 & 0.19 & 0.20 & 0.19 \\
\hline $3-26$ & 0.0 & 0.03 & 0.06 & 0.03 & 0.06 & 0.04 & 0.02 \\
\hline $3-29$ & 0.0 & -0.05 & -0.05 & 0.04 & 0.05 & 0.04 & 0.01 \\
\hline $3-35$ & -0.02 & -0.02 & 0.19 & -0.04 & 0.01 & 0.0 & 0.0 \\
\hline $3-37$ & -0.05 & -0.05 & 0.03 & -0.01 & -0.03 & 0.02 & -0.05 \\
\hline $3-51$ & 0.0 & -0.07 & 0.06 & 0.14 & 0.12 & -0.14 & -0.07 \\
\hline $3-55$ & 0.01 & -0.07 & 0.09 & -0.01 & 0.03 & -0.02 & 0.0 \\
\hline $3-56$ & 0.02 & -0.06 & 0.06 & -0.02 & 0.02 & 0.0 & 0.02 \\
\hline $3-57$ & 0.0 & -0.03 & 0.02 & 0.02 & 0.0 & 0.05 & 0.0 \\
\hline $4-22$ & 0.21 & 0.21 & 0.21 & 0.20 & 0.21 & 0.20 & 0.21 \\
\hline $4-23$ & 0.25 & 0.25 & 0.26 & 0.26 & 0.25 & 0.25 & 0.25 \\
\hline $4-30$ & -0.29 & -0.30 & -0.30 & -0.30 & -0.29 & -0.29 & -0.29 \\
\hline $5-7$ & 0.05 & 0.10 & 0.10 & 0.05 & 0.08 & 0.02 & 0.02 \\
\hline $5-8$ & -0.03 & 0.07 & 0.05 & 0.09 & 0.12 & 0.12 & 0.09 \\
\hline
\end{tabular}


TABLE 3 (continued)

\begin{tabular}{|c|c|c|c|c|c|c|c|}
\hline Term & $\mathrm{C}_{5}$ & $\mathrm{C}_{7}^{\mathrm{eq}}$ & $\mathrm{C}_{7}^{\mathrm{ax}}$ & $\beta_{2}$ & $\alpha_{\mathrm{R}}$ & $\alpha_{\mathrm{L}}$ & $\alpha^{\prime}$ \\
\hline $5-9$ & 0.17 & 0.32 & 0.38 & 0.18 & 0.21 & 0.21 & 0.15 \\
\hline $5-14$ & 0.25 & 0.16 & 0.25 & 0.21 & 0.16 & 0.24 & 0.24 \\
\hline $5-15$ & 0.11 & 0.08 & 0.03 & 0.05 & 0.07 & 0.05 & 0.09 \\
\hline $5-22$ & 0.02 & -0.03 & -0.08 & 0.02 & 0.03 & 0.0 & 0.02 \\
\hline $5-23$ & 0.06 & 0.0 & -0.13 & 0.07 & 0.08 & 0.03 & 0.06 \\
\hline $5-24$ & 0.26 & 0.42 & 0.60 & 0.26 & 0.22 & 0.32 & 0.27 \\
\hline $5-25$ & -0.18 & -0.18 & -0.20 & -0.18 & -0.18 & -0.18 & -0.20 \\
\hline $5-26$ & 0.11 & 0.11 & 0.20 & -0.04 & -0.05 & -0.04 & -0.05 \\
\hline $5-27$ & -0.10 & 0.04 & 0.07 & 0.09 & 0.11 & 0.09 & 0.08 \\
\hline $5-35$ & 0.17 & 0.06 & 0.67 & 0.14 & 0.16 & 0.29 & 0.16 \\
\hline $5-36$ & -0.16 & -0.24 & -0.09 & -0.17 & -0.15 & -0.17 & -0.16 \\
\hline $5-37$ & 0.31 & 0.31 & 0.29 & 0.30 & 0.31 & 0.29 & 0.31 \\
\hline $5-38$ & 0.21 & 0.46 & 0.32 & 0.23 & 0.24 & 0.23 & 0.19 \\
\hline $5-39$ & 0.38 & 0.30 & 0.35 & 0.32 & 0.31 & 0.35 & 0.36 \\
\hline $5-43$ & -0.05 & -0.04 & 0.0 & -0.05 & -0.04 & -0.01 & -0.06 \\
\hline $5-44$ & 0.07 & 0.07 & 0.06 & 0.08 & 0.06 & 0.07 & 0.06 \\
\hline $5-51$ & 0.0 & -0.07 & 0.08 & 0.05 & 0.09 & -0.08 & -0.03 \\
\hline $5-52$ & 0.02 & 0.05 & -0.01 & 0.05 & 0.0 & 0.0 & -0.05 \\
\hline $5-53$ & 0.0 & 0.05 & -0.06 & 0.02 & 0.0 & 0.0 & -0.03 \\
\hline 5-56 & 0.07 & -0.19 & 0.16 & 0.0 & -0.02 & -0.06 & 0.07 \\
\hline $5-57$ & 0.04 & 0.17 & -0.19 & 0.03 & 0.02 & 0.0 & -0.02 \\
\hline $5-58$ & 0.0 & 0.09 & -0.10 & 0.04 & 0.0 & 0.03 & -0.05 \\
\hline $6-7$ & 0.0 & 0.02 & 0.01 & 0.04 & 0.04 & 0.06 & 0.0 \\
\hline $6-8$ & -0.01 & 0.05 & 0.07 & 0.0 & 0.01 & 0.0 & -0.02 \\
\hline $6-9$ & 0.0 & 0.0 & 0.0 & -0.05 & -0.07 & -0.04 & -0.02 \\
\hline $6-10$ & -0.07 & -0.05 & -0.03 & -0.05 & -0.05 & -0.05 & -0.08 \\
\hline $6-26$ & -0.05 & 0.05 & 0.05 & -0.07 & -0.09 & -0.06 & -0.07 \\
\hline $6-28$ & -0.09 & -0.07 & -0.06 & -0.09 & -0.09 & -0.10 & -0.08 \\
\hline $6-29$ & 0.04 & 0.11 & 0.12 & 0.07 & 0.07 & 0.06 & 0.03 \\
\hline $6-38$ & 0.0 & 0.0 & 0.0 & -0.07 & -0.09 & -0.06 & 0.01 \\
\hline $6-53$ & 0.01 & 0.06 & -0.04 & 0.0 & -0.02 & 0.02 & 0.03 \\
\hline $6-57$ & 0.01 & 0.05 & -0.04 & -0.02 & 0.02 & -0.04 & -0.02 \\
\hline $7-8$ & 1.31 & 1.30 & 1.28 & 1.32 & 1.34 & 1.32 & 1.30 \\
\hline $7-9$ & 0.41 & 0.37 & 0.40 & 0.36 & 0.41 & 0.38 & 0.34 \\
\hline $7-10$ & -0.08 & -0.07 & -0.07 & -0.07 & -0.07 & -0.07 & -0.06 \\
\hline $7-25$ & -0.06 & -0.01 & 0.0 & 0.0 & 0.0 & -0.01 & 0.01 \\
\hline $7-26$ & -0.54 & -0.47 & -0.47 & -0.50 & -0.49 & -0.51 & -0.50 \\
\hline $7-27$ & -0.05 & -0.09 & -0.09 & -0.11 & -0.09 & -0.11 & -0.12 \\
\hline $7-35$ & 0.03 & -0.02 & 0.12 & 0.02 & 0.06 & -0.01 & 0.05 \\
\hline $7-37$ & -0.04 & -0.07 & 0.0 & -0.05 & -0.03 & -0.04 & 0.0 \\
\hline $7-38$ & 0.03 & 0.10 & 0.08 & 0.10 & 0.11 & 0.08 & 0.03 \\
\hline $7-39$ & -0.04 & 0.0 & -0.08 & 0.0 & -0.04 & -0.01 & -0.07 \\
\hline $7-55$ & 0.0 & -0.05 & 0.05 & -0.01 & -0.01 & 0.01 & 0.0 \\
\hline $7-56$ & 0.0 & -0.06 & 0.06 & -0.04 & 0.0 & -0.02 & 0.02 \\
\hline
\end{tabular}


TABLE 3 (continued)

\begin{tabular}{|c|c|c|c|c|c|c|c|}
\hline Term & $\mathrm{C}_{5}$ & $\mathrm{C}_{7}^{\text {eq }}$ & $\mathrm{C}_{7}^{\mathrm{ax}}$ & $\beta_{2}$ & $\alpha_{\mathrm{R}}$ & $\alpha_{\mathrm{L}}$ & $\alpha^{\prime}$ \\
\hline $8-9$ & 0.24 & 0.23 & 0.19 & 0.26 & 0.19 & 0.27 & 0.31 \\
\hline $8-10$ & 0.10 & 0.11 & 0.11 & 0.09 & 0.10 & 0.09 & 0.10 \\
\hline $8-21$ & -0.05 & -0.05 & -0.05 & -0.04 & -0.05 & -0.04 & -0.05 \\
\hline $8-22$ & 0.0 & 0.04 & 0.06 & 0.0 & 0.0 & 0.0 & 0.0 \\
\hline $8-23$ & 0.0 & 0.05 & 0.08 & 0.0 & 0.0 & -0.01 & -0.02 \\
\hline $8-24$ & 0.03 & -0.05 & -0.09 & 0.03 & 0.02 & 0.04 & 0.0 \\
\hline $8-26$ & 0.18 & 0.14 & 0.08 & 0.14 & 0.09 & 0.15 & 0.20 \\
\hline $8-27$ & -0.45 & -0.42 & -0.42 & -0.44 & -0.44 & -0.44 & -0.45 \\
\hline $8-28$ & 0.18 & 0.15 & 0.17 & 0.17 & 0.19 & 0.18 & 0.18 \\
\hline $8-29$ & 0.19 & 0.25 & 0.26 & 0.18 & 0.17 & 0.18 & 0.19 \\
\hline $8-35$ & -0.02 & 0.05 & -0.10 & 0.02 & -0.01 & 0.04 & 0.05 \\
\hline $8-36$ & 0.0 & 0.0 & -0.06 & -0.02 & -0.03 & -0.01 & -0.02 \\
\hline $8-37$ & -0.04 & 0.0 & -0.07 & 0.0 & -0.02 & -0.02 & -0.05 \\
\hline $8-38$ & -0.02 & -0.04 & -0.04 & -0.02 & -0.03 & 0.0 & 0.06 \\
\hline $8-39$ & -0.05 & -0.08 & 0.0 & -0.06 & -0.03 & -0.05 & -0.01 \\
\hline $8-48$ & 0.07 & 0.07 & 0.08 & 0.07 & 0.06 & 0.07 & 0.06 \\
\hline $8-50$ & 0.0 & -0.06 & 0.07 & -0.01 & -0.01 & 0.02 & 0.0 \\
\hline $8-52$ & 0.03 & 0.0 & -0.04 & 0.0 & -0.04 & 0.07 & 0.04 \\
\hline $8-53$ & 0.01 & 0.08 & -0.06 & 0.0 & -0.09 & 0.08 & 0.09 \\
\hline $8-55$ & 0.0 & 0.10 & -0.11 & 0.01 & 0.01 & -0.02 & -0.02 \\
\hline $8-56$ & -0.01 & 0.13 & -0.17 & 0.03 & 0.0 & 0.0 & -0.06 \\
\hline $8-57$ & -0.02 & 0.02 & 0.0 & 0.0 & 0.02 & -0.05 & -0.06 \\
\hline $9-14$ & 0.19 & 0.18 & 0.17 & 0.19 & 0.19 & 0.19 & 0.18 \\
\hline $9-16$ & 0.0 & -0.06 & 0.0 & -0.02 & 0.0 & -0.02 & -0.01 \\
\hline $9-17$ & 0.0 & -0.02 & 0.0 & -0.06 & -0.05 & -0.05 & 0.0 \\
\hline $9-23$ & -0.02 & -0.08 & -0.13 & 0.0 & 0.0 & 0.01 & 0.0 \\
\hline $9-24$ & 0.0 & 0.18 & 0.29 & 0.0 & -0.01 & 0.0 & 0.01 \\
\hline $9-26$ & 0.11 & 0.36 & 0.46 & 0.31 & 0.38 & 0.31 & 0.29 \\
\hline $9-27$ & 0.43 & 0.29 & 0.22 & 0.31 & 0.24 & 0.31 & 0.24 \\
\hline $9-29$ & 0.02 & 0.04 & 0.06 & 0.02 & 0.02 & 0.02 & 0.02 \\
\hline $9-35$ & 0.28 & 0.10 & 0.40 & 0.23 & 0.20 & 0.26 & 0.17 \\
\hline $9-36$ & -0.10 & -0.19 & -0.05 & -0.11 & -0.12 & -0.10 & -0.13 \\
\hline $9-37$ & -0.18 & -0.19 & -0.17 & -0.17 & -0.19 & -0.16 & -0.18 \\
\hline $9-38$ & 0.47 & 0.19 & 0.23 & 0.15 & 0.24 & 0.17 & 0.17 \\
\hline $9-39$ & -0.20 & -0.31 & -0.18 & -0.36 & -0.26 & -0.35 & -0.17 \\
\hline $9-50$ & 0.0 & 0.06 & -0.06 & 0.0 & 0.0 & 0.0 & 0.0 \\
\hline $9-51$ & 0.0 & -0.10 & 0.13 & -0.03 & -0.05 & 0.04 & 0.0 \\
\hline $9-52$ & 0.0 & -0.05 & 0.05 & -0.07 & 0.0 & -0.07 & 0.03 \\
\hline $9-55$ & 0.0 & -0.11 & 0.12 & 0.0 & 0.0 & 0.0 & 0.0 \\
\hline $9-56$ & -0.03 & -0.24 & 0.27 & -0.04 & -0.02 & -0.03 & -0.02 \\
\hline $9-57$ & -0.06 & 0.06 & -0.05 & 0.0 & -0.06 & 0.0 & 0.02 \\
\hline $10-20$ & 0.10 & 0.11 & 0.13 & 0.09 & 0.13 & 0.10 & 0.10 \\
\hline $10-21$ & 0.10 & 0.13 & 0.11 & 0.12 & 0.08 & 0.12 & 0.11 \\
\hline $10-26$ & 0.07 & 0.07 & 0.07 & 0.08 & 0.08 & 0.08 & 0.09 \\
\hline $10-27$ & 0.10 & 0.10 & 0.10 & 0.10 & 0.10 & 0.10 & 0.09 \\
\hline $10-28$ & 0.21 & 0.23 & 0.24 & 0.22 & 0.23 & 0.22 & 0.22 \\
\hline $10-29$ & -0.18 & -0.18 & -0.19 & -0.18 & -0.18 & -0.18 & -0.18 \\
\hline
\end{tabular}


TABLE 3 (continued)

\begin{tabular}{|c|c|c|c|c|c|c|c|}
\hline Term & $\mathrm{C}_{5}$ & $\mathrm{C}_{7}^{\mathrm{eq}}$ & $C_{7}^{a x}$ & $\beta_{2}$ & $\alpha_{R}$ & $\alpha_{\mathrm{L}}$ & $\alpha^{\prime}$ \\
\hline $10-45$ & -0.51 & -0.50 & -0.50 & -0.50 & -0.50 & -0.50 & -0.51 \\
\hline $10-48$ & -0.05 & -0.05 & -0.04 & -0.05 & -0.05 & -0.05 & -0.05 \\
\hline $11-22$ & 0.06 & 0.06 & 0.06 & 0.06 & 0.06 & 0.06 & 0.06 \\
\hline $11-23$ & -0.05 & -0.05 & -0.05 & -0.05 & -0.05 & -0.05 & -0.05 \\
\hline $11-31$ & -0.10 & -0.10 & -0.09 & -0.10 & -0.09 & -0.09 & -0.09 \\
\hline $11-33$ & 0.10 & 0.10 & 0.10 & 0.10 & 0.10 & 0.10 & 0.10 \\
\hline $12-30$ & 0.05 & 0.06 & 0.05 & 0.05 & 0.05 & 0.06 & 0.05 \\
\hline $12-31$ & 0.06 & 0.05 & 0.06 & 0.06 & 0.06 & 0.05 & 0.06 \\
\hline $12-32$ & 0.10 & 0.09 & 0.10 & 0.10 & 0.10 & 0.10 & 0.10 \\
\hline $12-33$ & -0.05 & -0.05 & -0.05 & -0.05 & -0.05 & -0.05 & -0.05 \\
\hline $12-34$ & 0.09 & 0.09 & 0.09 & 0.09 & 0.09 & 0.09 & 0.09 \\
\hline $13-30$ & 0.06 & 0.05 & 0.06 & 0.06 & 0.06 & 0.05 & 0.05 \\
\hline $13-31$ & 0.06 & 0.06 & 0.05 & 0.05 & 0.06 & 0.05 & 0.05 \\
\hline $13-32$ & -0.09 & -0.10 & -0.10 & -0.09 & -0.10 & -0.10 & -0.10 \\
\hline $13-33$ & -0.05 & -0.05 & -0.05 & -0.05 & -0.05 & -0.05 & -0.05 \\
\hline $13-34$ & -0.09 & -0.09 & -0.09 & -0.09 & -0.09 & -0.09 & -0.09 \\
\hline $14-24$ & 0.02 & 0.04 & 0.06 & 0.01 & 0.04 & 0.04 & 0.01 \\
\hline $14-26$ & 0.02 & 0.08 & 0.02 & 0.07 & 0.04 & 0.06 & 0.02 \\
\hline $14-35$ & 0.10 & 0.15 & 0.16 & 0.13 & 0.15 & 0.16 & 0.14 \\
\hline $14-36$ & 0.23 & 0.20 & 0.22 & 0.21 & 0.22 & 0.21 & 0.22 \\
\hline $14-38$ & -0.25 & -0.26 & -0.24 & -0.26 & -0.29 & -0.28 & -0.23 \\
\hline $14-39$ & -0.07 & -0.07 & -0.02 & -0.11 & -0.07 & -0.11 & -0.05 \\
\hline $14-40$ & -0.33 & -0.33 & -0.35 & -0.33 & -0.33 & -0.34 & -0.33 \\
\hline $15-23$ & 0.01 & 0.03 & 0.01 & 0.05 & 0.04 & 0.0 & 0.02 \\
\hline $15-24$ & -0.05 & -0.07 & 0.0 & -0.09 & -0.07 & 0.02 & -0.04 \\
\hline $15-26$ & -0.04 & -0.05 & 0.07 & -0.02 & 0.02 & -0.02 & -0.05 \\
\hline $15-27$ & 0.02 & 0.02 & -0.06 & 0.0 & -0.03 & 0.02 & -0.05 \\
\hline $15-35$ & -0.12 & -0.12 & -0.11 & -0.14 & -0.12 & -0.15 & -0.11 \\
\hline $16-24$ & 0.0 & 0.0 & -0.09 & 0.0 & 0.0 & -0.05 & 0.0 \\
\hline $16-35$ & -0.04 & -0.06 & -0.12 & -0.04 & -0.03 & -0.09 & -0.08 \\
\hline $16-38$ & 0.01 & 0.02 & 0.05 & 0.0 & 0.0 & 0.03 & 0.02 \\
\hline $16-40$ & 0.06 & 0.06 & 0.06 & 0.07 & 0.06 & 0.05 & 0.08 \\
\hline $16-41$ & -0.11 & -0.11 & -0.09 & -0.12 & -0.12 & -0.10 & -0.12 \\
\hline $16-43$ & 0.08 & 0.09 & 0.06 & 0.09 & 0.10 & 0.08 & 0.07 \\
\hline $16-56$ & 0.0 & 0.0 & 0.08 & 0.0 & 0.0 & 0.05 & 0.0 \\
\hline $17-39$ & 0.08 & 0.05 & 0.04 & 0.10 & 0.08 & 0.10 & 0.05 \\
\hline $17-40$ & 0.07 & 0.06 & 0.05 & 0.05 & 0.05 & 0.06 & 0.06 \\
\hline $17-41$ & 0.06 & 0.05 & 0.05 & 0.05 & 0.05 & 0.05 & 0.05 \\
\hline $17-42$ & 0.10 & 0.10 & 0.10 & 0.09 & 0.08 & 0.09 & 0.10 \\
\hline $17-43$ & -0.04 & -0.05 & -0.05 & -0.05 & -0.05 & -0.05 & -0.05 \\
\hline $17-44$ & 0.07 & 0.08 & 0.09 & 0.08 & 0.08 & 0.07 & 0.09 \\
\hline $18-39$ & -0.07 & -0.05 & -0.04 & -0.05 & -0.05 & -0.05 & -0.07 \\
\hline $18-40$ & 0.05 & 0.06 & 0.07 & 0.06 & 0.06 & 0.06 & 0.05 \\
\hline $18-41$ & 0.05 & 0.05 & 0.05 & 0.05 & 0.05 & 0.06 & 0.05 \\
\hline $18-42$ & -0.08 & -0.09 & -0.10 & -0.09 & -0.10 & -0.10 & -0.08 \\
\hline $18-44$ & -0.07 & -0.08 & -0.07 & -0.08 & -0.08 & -0.08 & -0.07 \\
\hline
\end{tabular}


TABLE 3 (continued)

\begin{tabular}{|c|c|c|c|c|c|c|c|}
\hline Term & $\mathrm{C}_{5}$ & $\mathrm{C}_{7}^{\mathrm{eq}}$ & $C_{7}^{a x}$ & $\beta_{2}$ & $\alpha_{\mathrm{R}}$ & $\alpha_{L}$ & $\alpha^{\prime}$ \\
\hline $19-45$ & 0.06 & 0.06 & 0.06 & 0.06 & 0.06 & 0.06 & 0.06 \\
\hline $19-46$ & -0.12 & -0.12 & -0.12 & -0.12 & -0.12 & -0.12 & -0.12 \\
\hline $19-48$ & 0.08 & 0.08 & 0.08 & 0.08 & 0.08 & 0.08 & 0.08 \\
\hline $20-45$ & 0.06 & 0.06 & 0.06 & 0.06 & 0.06 & 0.06 & 0.06 \\
\hline $20-46$ & 0.06 & 0.06 & 0.07 & 0.06 & 0.06 & 0.07 & 0.06 \\
\hline $20-47$ & 0.10 & 0.10 & 0.10 & 0.09 & 0.10 & 0.10 & 0.10 \\
\hline $20-49$ & 0.05 & 0.05 & 0.05 & 0.05 & 0.05 & 0.05 & 0.05 \\
\hline $21-28$ & -0.04 & -0.03 & -0.04 & -0.03 & -0.05 & -0.03 & -0.03 \\
\hline $21-45$ & 0.06 & 0.06 & 0.06 & 0.06 & 0.05 & 0.06 & 0.06 \\
\hline $21-46$ & 0.06 & 0.07 & 0.06 & 0.06 & 0.06 & 0.06 & 0.06 \\
\hline $21-47$ & -0.10 & -0.10 & -0.10 & -0.10 & -0.09 & -0.10 & -0.10 \\
\hline $21-49$ & -0.05 & -0.05 & -0.05 & -0.05 & -0.06 & -0.05 & -0.05 \\
\hline $22-23$ & 0.16 & 0.19 & 0.20 & 0.17 & 0.15 & 0.15 & 0.17 \\
\hline $22-24$ & 0.04 & -0.02 & -0.09 & 0.0 & 0.03 & 0.03 & 0.02 \\
\hline $22-26$ & 0.02 & -0.03 & -0.06 & 0.0 & 0.0 & 0.0 & 0.0 \\
\hline $22-33$ & 0.12 & 0.12 & 0.11 & 0.12 & 0.12 & 0.12 & 0.12 \\
\hline $22-35$ & 0.01 & 0.05 & -0.19 & 0.03 & 0.03 & -0.06 & 0.03 \\
\hline $22-38$ & 0.02 & -0.06 & -0.02 & 0.02 & 0.0 & 0.0 & 0.04 \\
\hline $23-24$ & -0.04 & -0.16 & -0.29 & -0.08 & -0.04 & -0.06 & -0.07 \\
\hline $23-25$ & -0.05 & -0.05 & -0.05 & -0.05 & -0.06 & -0.06 & -0.05 \\
\hline $23-26$ & 0.01 & -0.09 & -0.14 & 0.0 & 0.0 & 0.0 & -0.01 \\
\hline $23-33$ & -0.09 & -0.08 & -0.08 & -0.08 & -0.08 & -0.08 & -0.08 \\
\hline $23-35$ & 0.01 & 0.08 & -0.36 & 0.04 & 0.03 & -0.10 & 0.03 \\
\hline $23-36$ & 0.0 & 0.04 & -0.05 & 0.0 & 0.0 & 0.0 & 0.0 \\
\hline $23-38$ & 0.02 & -0.15 & -0.08 & 0.02 & 0.0 & 0.0 & 0.04 \\
\hline $23-51$ & -0.02 & 0.06 & -0.07 & 0.0 & 0.0 & 0.01 & -0.01 \\
\hline $23-55$ & -0.02 & 0.06 & -0.05 & 0.0 & -0.02 & 0.03 & -0.02 \\
\hline $23-56$ & -0.04 & 0.13 & -0.13 & 0.03 & -0.01 & 0.02 & -0.05 \\
\hline $23-57$ & 0.0 & -0.05 & 0.06 & -0.01 & -0.01 & 0.01 & 0.0 \\
\hline 24-26 & 0.04 & 0.20 & 0.35 & -0.02 & 0.0 & 0.0 & -0.02 \\
\hline $24-28$ & -0.01 & -0.03 & -0.05 & 0.0 & 0.0 & 0.0 & 0.02 \\
\hline $24-35$ & -0.02 & -0.12 & 0.78 & -0.03 & -0.02 & 0.20 & -0.04 \\
\hline $24-36$ & 0.02 & -0.10 & 0.10 & -0.02 & 0.0 & -0.03 & 0.02 \\
\hline $24-38$ & -0.05 & 0.33 & 0.21 & 0.0 & 0.0 & 0.0 & -0.05 \\
\hline $24-39$ & 0.09 & 0.04 & 0.08 & 0.02 & 0.03 & 0.06 & 0.09 \\
\hline $24-43$ & 0.0 & 0.0 & 0.06 & 0.0 & 0.0 & 0.04 & 0.0 \\
\hline $24-51$ & 0.03 & -0.11 & 0.12 & 0.04 & 0.05 & -0.08 & 0.0 \\
\hline $24-52$ & 0.02 & -0.06 & 0.08 & 0.04 & 0.03 & -0.07 & -0.05 \\
\hline $24-53$ & 0.0 & 0.07 & -0.10 & 0.0 & 0.0 & 0.0 & 0.02 \\
\hline $24-55$ & 0.03 & -0.06 & 0.04 & -0.02 & 0.0 & -0.03 & 0.04 \\
\hline $24-56$ & 0.07 & -0.22 & 0.21 & -0.05 & 0.04 & -0.07 & 0.07 \\
\hline $24-57$ & 0.03 & 0.24 & -0.31 & 0.07 & 0.09 & -0.10 & 0.02 \\
\hline $24-58$ & 0.02 & 0.08 & -0.11 & 0.04 & 0.02 & -0.02 & 0.0 \\
\hline $26-27$ & 0.02 & 0.04 & 0.06 & 0.06 & 0.09 & 0.07 & 0.13 \\
\hline $26-29$ & -0.01 & 0.05 & 0.07 & -0.02 & -0.03 & -0.03 & -0.02 \\
\hline $26-35$ & 0.0 & -0.04 & 0.40 & 0.04 & 0.09 & 0.02 & 0.07 \\
\hline $26-36$ & 0.04 & -0.08 & 0.04 & -0.03 & -0.03 & -0.03 & -0.05 \\
\hline $26-37$ & 0.0 & 0.0 & -0.06 & 0.02 & -0.06 & 0.03 & -0.08 \\
\hline
\end{tabular}


TABLE 3 (continued)

\begin{tabular}{|c|c|c|c|c|c|c|c|}
\hline Term & $\mathrm{C}_{5}$ & $\mathrm{C}_{7}^{\text {eq }}$ & $\mathrm{C}_{7}^{\operatorname{ax}}$ & $\beta_{2}$ & $\alpha_{\mathrm{R}}$ & $\alpha_{\mathrm{L}}$ & $\alpha^{\prime}$ \\
\hline $26-38$ & 0.0 & 0.23 & 0.21 & 0.15 & 0.20 & 0.12 & 0.0 \\
\hline $26-39$ & -0.06 & -0.02 & -0.03 & -0.03 & 0.0 & -0.04 & 0.0 \\
\hline $26-50$ & 0.0 & 0.07 & -0.09 & 0.0 & 0.0 & 0.0 & -0.01 \\
\hline $26-51$ & 0.0 & -0.11 & 0.15 & -0.04 & -0.07 & 0.04 & 0.02 \\
\hline $26-55$ & -0.01 & -0.10 & 0.12 & 0.01 & 0.03 & -0.03 & 0.01 \\
\hline $26-56$ & -0.02 & -0.26 & 0.34 & -0.05 & -0.02 & -0.02 & 0.02 \\
\hline $26-57$ & -0.01 & 0.05 & -0.09 & 0.07 & -0.05 & 0.10 & -0.02 \\
\hline $27-28$ & 0.04 & 0.05 & 0.05 & 0.04 & 0.04 & 0.04 & 0.03 \\
\hline $27-29$ & -0.06 & -0.06 & -0.06 & -0.06 & -0.07 & -0.06 & -0.06 \\
\hline $27-35$ & 0.09 & 0.06 & 0.0 & 0.05 & 0.03 & 0.06 & 0.04 \\
\hline $27-38$ & 0.16 & -0.05 & 0.02 & -0.01 & 0.03 & 0.0 & 0.05 \\
\hline $27-39$ & -0.02 & -0.07 & -0.04 & -0.09 & -0.05 & -0.08 & -0.04 \\
\hline $27-56$ & 0.0 & 0.06 & -0.06 & 0.02 & 0.01 & -0.01 & -0.04 \\
\hline $28-29$ & -0.02 & -0.07 & -0.08 & -0.02 & -0.02 & -0.02 & -0.02 \\
\hline $28-35$ & 0.03 & 0.04 & -0.12 & 0.0 & -0.04 & 0.0 & -0.05 \\
\hline $28-38$ & 0.05 & -0.07 & -0.05 & -0.06 & -0.08 & -0.07 & -0.01 \\
\hline $28-56$ & 0.01 & 0.06 & -0.07 & 0.02 & 0.0 & 0.02 & -0.03 \\
\hline $29-35$ & 0.0 & 0.0 & 0.07 & 0.0 & 0.0 & 0.0 & 0.02 \\
\hline $34-50$ & 0.08 & 0.08 & 0.08 & 0.08 & 0.08 & 0.08 & 0.08 \\
\hline $35-36$ & 0.03 & 0.07 & 0.18 & 0.04 & 0.06 & 0.02 & 0.03 \\
\hline $35-37$ & -0.06 & -0.05 & -0.04 & -0.08 & -0.08 & -0.09 & -0.05 \\
\hline $35-38$ & 0.27 & -0.19 & 0.31 & 0.03 & 0.15 & 0.02 & 0.10 \\
\hline $35-39$ & 0.06 & -0.06 & 0.04 & -0.04 & 0.02 & 0.0 & -0.01 \\
\hline $35-43$ & -0.08 & -0.06 & -0.02 & -0.08 & -0.07 & -0.05 & -0.06 \\
\hline $35-50$ & 0.0 & -0.03 & -0.08 & -0.01 & -0.01 & 0.0 & 0.0 \\
\hline $35-51$ & 0.03 & 0.01 & 0.19 & 0.0 & -0.05 & -0.03 & 0.05 \\
\hline $35-52$ & -0.03 & 0.05 & 0.08 & 0.01 & 0.03 & -0.03 & 0.0 \\
\hline $35-53$ & -0.02 & -0.02 & -0.05 & 0.02 & 0.0 & 0.0 & -0.02 \\
\hline $35-55$ & 0.0 & 0.07 & 0.11 & 0.01 & 0.04 & -0.02 & 0.0 \\
\hline $35-56$ & 0.0 & 0.15 & 0.38 & 0.03 & 0.06 & -0.04 & -0.04 \\
\hline $35-57$ & -0.08 & -0.04 & -0.21 & -0.02 & -0.07 & -0.02 & -0.04 \\
\hline $35-58$ & 0.0 & -0.05 & -0.05 & -0.04 & -0.01 & -0.01 & 0.0 \\
\hline $36-38$ & -0.03 & -0.14 & 0.11 & -0.04 & 0.0 & -0.02 & 0.0 \\
\hline $36-43$ & 0.07 & 0.07 & 0.06 & 0.07 & 0.07 & 0.07 & 0.06 \\
\hline $36-51$ & 0.04 & 0.04 & 0.06 & 0.02 & 0.0 & 0.0 & 0.05 \\
\hline $36-55$ & 0.0 & 0.05 & 0.08 & -0.02 & 0.02 & 0.04 & 0.0 \\
\hline $36-56$ & 0.05 & 0.14 & 0.18 & 0.02 & 0.06 & 0.07 & 0.04 \\
\hline $36-57$ & -0.05 & -0.06 & -0.09 & -0.05 & -0.04 & -0.05 & -0.05 \\
\hline $37-55$ & 0.03 & 0.05 & 0.07 & 0.02 & 0.0 & 0.04 & 0.03 \\
\hline $37-56$ & 0.11 & 0.06 & 0.12 & 0.05 & 0.02 & 0.10 & 0.11 \\
\hline $37-57$ & 0.05 & 0.09 & 0.09 & 0.06 & 0.09 & 0.10 & 0.07 \\
\hline $37-58$ & 0.0 & 0.05 & 0.05 & 0.02 & 0.02 & 0.02 & 0.02 \\
\hline $38-39$ & -0.06 & 0.03 & -0.14 & 0.05 & 0.0 & 0.03 & -0.09 \\
\hline $38-43$ & 0.06 & 0.04 & 0.02 & 0.05 & 0.05 & 0.03 & 0.05 \\
\hline $38-44$ & -0.02 & -0.04 & -0.03 & -0.05 & -0.03 & -0.05 & -0.02 \\
\hline $38-50$ & -0.01 & 0.06 & -0.08 & -0.02 & -0.02 & 0.0 & 0.0 \\
\hline $38-51$ & -0.05 & -0.20 & 0.22 & -0.08 & -0.12 & 0.13 & -0.05 \\
\hline
\end{tabular}


TABLE 3 (continued)

\begin{tabular}{|c|c|c|c|c|c|c|c|}
\hline Term & $\mathrm{C}_{5}$ & $\mathrm{C}_{7}^{\mathrm{eq}}$ & $C_{7}^{a x}$ & $\beta_{2}$ & $\alpha_{\mathrm{R}}$ & $\alpha_{\mathrm{L}}$ & $\alpha^{\prime}$ \\
\hline $38-52$ & -0.01 & -0.03 & 0.02 & -0.05 & 0.03 & -0.08 & 0.0 \\
\hline 38-55 & 0.0 & -0.08 & 0.10 & 0.04 & 0.06 & -0.03 & 0.0 \\
\hline $38-56$ & -0.05 & -0.36 & 0.34 & -0.06 & -0.02 & 0.03 & -0.05 \\
\hline $38-57$ & 0.02 & 0.17 & -0.17 & 0.12 & 0.02 & 0.10 & -0.08 \\
\hline $39-44$ & 0.10 & 0.11 & 0.12 & 0.10 & 0.12 & 0.09 & 0.13 \\
\hline $39-50$ & 0.0 & 0.04 & 0.05 & 0.03 & 0.02 & 0.02 & 0.0 \\
\hline $39-51$ & 0.07 & 0.0 & -0.08 & 0.02 & 0.01 & -0.07 & 0.05 \\
\hline $39-55$ & 0.0 & -0.05 & -0.10 & -0.03 & -0.02 & -0.04 & 0.01 \\
\hline $39-56$ & 0.01 & -0.07 & -0.16 & -0.04 & -0.04 & -0.10 & 0.02 \\
\hline $39-57$ & 0.02 & -0.08 & -0.12 & -0.02 & -0.04 & -0.06 & -0.11 \\
\hline $39-58$ & 0.01 & -0.04 & -0.06 & 0.0 & -0.02 & -0.02 & -0.02 \\
\hline $49-59$ & 0.05 & 0.05 & 0.05 & 0.05 & 0.05 & 0.05 & 0.06 \\
\hline $50-51$ & -0.05 & -0.08 & -0.13 & -0.05 & -0.05 & -0.07 & -0.06 \\
\hline $50-55$ & -0.08 & -0.14 & -0.15 & -0.06 & -0.07 & -0.10 & -0.09 \\
\hline $50-56$ & -0.10 & -0.18 & -0.24 & -0.08 & -0.08 & -0.12 & -0.12 \\
\hline $50-57$ & 0.0 & -0.05 & -0.07 & -0.01 & -0.02 & -0.04 & 0.0 \\
\hline $51-55$ & -0.08 & -0.02 & 0.05 & -0.07 & -0.07 & -0.04 & -0.07 \\
\hline 51-56 & 0.0 & 0.16 & 0.25 & 0.02 & 0.05 & 0.14 & 0.08 \\
\hline $51-57$ & -0.10 & -0.02 & -0.02 & -0.05 & 0.03 & 0.09 & -0.08 \\
\hline $52-53$ & -0.07 & -0.07 & -0.09 & -0.07 & -0.05 & -0.05 & -0.07 \\
\hline $52-55$ & 0.02 & -0.06 & -0.05 & -0.05 & -0.05 & -0.04 & 0.0 \\
\hline $52-56$ & 0.10 & 0.02 & 0.02 & -0.08 & -0.11 & -0.13 & -0.07 \\
\hline $52-57$ & -0.01 & -0.08 & -0.10 & 0.0 & 0.08 & -0.04 & -0.09 \\
\hline $53-55$ & 0.02 & 0.08 & 0.09 & 0.04 & 0.01 & 0.0 & 0.02 \\
\hline $53-56$ & -0.02 & 0.07 & 0.09 & 0.07 & 0.06 & 0.04 & 0.04 \\
\hline $53-57$ & 0.06 & 0.15 & 0.19 & 0.07 & 0.06 & 0.06 & 0.07 \\
\hline $53-58$ & -0.09 & 0.0 & 0.0 & -0.07 & -0.06 & -0.05 & -0.08 \\
\hline $55-56$ & 0.13 & 0.29 & 0.39 & 0.10 & 0.13 & 0.20 & 0.14 \\
\hline $55-57$ & 0.09 & 0.08 & 0.09 & 0.07 & 0.04 & 0.03 & 0.09 \\
\hline $56-57$ & 0.10 & 0.05 & 0.06 & 0.07 & 0.15 & 0.17 & 0.09 \\
\hline $56-58$ & 0.04 & 0.06 & 0.06 & -0.03 & -0.01 & 0.01 & 0.0 \\
\hline $57-58$ & 0.03 & 0.17 & 0.24 & 0.07 & 0.11 & 0.08 & 0.09 \\
\hline
\end{tabular}

'Units: energy in mdyn $\AA$, stretching coordinates in $\AA$, bending coordinates in radians. Group coordinates are defined in ref. 2.

$\left(\mathrm{C}_{5}\right)$ or indirectly through the adjacent $\mathrm{NH}\left(\mathrm{C}_{7}\right)$. There also seems to be an inverse relationship between the $\mathrm{CO}$ stretch and $\mathrm{CN}$ stretch force constants of each peptide unit; this is demonstrated in Fig. 2(b) and can be attributed to the electronic resonance in the peptide group. The points for $\mathrm{f}(\mathrm{CO} \operatorname{str} 1)$ versus $\mathrm{f}(\mathrm{CN}$ str 1$)$ in $\mathrm{C}_{7}^{\text {eq }}$ and $\mathrm{C}_{7}^{\mathrm{ax}}$ are exceptional probably because of the strong hydrogen bonds; that is, the $\mathrm{CO}$ bond is expected to be more affected than the $\mathrm{CN}$ bond by hydrogen bonding.

The changes in $\mathrm{f}(\mathrm{CO}$ str1) and $\mathrm{f}(\mathrm{CO}$ str2) also seem to be related to the planarity of the peptide groups. Figure 2 (c) shows a tendency for these terms 
TABLE 4

Normal modes of Ala dipeptides using scaled force constants

$\nu\left(\mathrm{cm}^{-1}\right) \underset{\left(\mathrm{km} \mathrm{mol}^{-1}\right)}{I^{\mathrm{a}}}$ Potential-energy distribution $>10 \%$

\begin{tabular}{|c|c|c|}
\hline$\beta_{2}$ & & \\
\hline 3455 & 39 & NH str2(98) \\
\hline 3431 & 40 & NH str1 (98) \\
\hline 3023 & 2 & MH1 str (86) \\
\hline 3010 & 3 & $\mathrm{CH} 14 \operatorname{str}(83), \mathrm{CH} 15 \operatorname{str}(14)$ \\
\hline 2996 & 3 & $\mathrm{C}^{\alpha} \mathrm{H}^{\alpha} \operatorname{str}(61), \mathrm{CH} 16 \operatorname{str}(19), \mathrm{CH} 15 \operatorname{str}(17)$ \\
\hline 2993 & 8 & MH22 str(65), MH20 (str(31) \\
\hline 2971 & 11 & MH21 str (49), MH20 str (38) \\
\hline 2968 & 3 & MH3 str(51), MH2 str (50) \\
\hline 2966 & 25 & $\mathrm{C}^{\alpha} \mathrm{H}^{\alpha} \operatorname{str}(36), \mathrm{CH} 15 \operatorname{str}(29), \mathrm{CH} 16 \operatorname{str}(26)$ \\
\hline 2904 & 1 & MH2 $\operatorname{str}(43), \mathrm{MH} 3 \operatorname{str}(39)$, MH1 $\operatorname{str}(14)$ \\
\hline 2902 & 15 & CH16 str(45), CH15 str(35), CH14 str (15) \\
\hline 2900 & 33 & MH21 str(43), MH20 str(28), MH22 str(27) \\
\hline 1719 & 211 & CO str1 (78) \\
\hline 1694 & 252 & CO str2(81) \\
\hline 1550 & 382 & NH ib2(53), CN str2(27) \\
\hline 1532 & 306 & NH ib1 (61), CN str1 (18) \\
\hline 1478 & 2 & M2 ab1 (92) \\
\hline 1466 & 6 & $\mathrm{C}^{\beta} \mathrm{ab2}(85)$ \\
\hline 1456 & 14 & $\mathrm{C}^{\beta} \mathrm{ab1}(89)$ \\
\hline 1455 & 6 & M2 ab2(95) \\
\hline 1447 & 12 & M1 ab2(93) \\
\hline 1435 & 26 & M1 ab1 (91) \\
\hline 1412 & 2 & $\mathrm{M} 2 \mathrm{sb}(103)$ \\
\hline 1379 & 9 & $\mathrm{C}^{\beta} \mathrm{sb}(104)$ \\
\hline 1371 & 32 & M1 sb(101) \\
\hline 1321 & 32 & $\mathrm{H}^{\alpha} \mathrm{b} 1(30), \mathrm{C}^{\alpha} \mathrm{C} \operatorname{str}(23), \mathrm{H}^{\alpha} \mathrm{b} 2(17), \mathrm{CN} \operatorname{str} 2(11)$ \\
\hline 1301 & 27 & $\mathrm{H}^{\alpha} \mathrm{b} 1(41), \mathrm{H}^{\alpha} \mathrm{b} 2(38)$ \\
\hline 1265 & 169 & CN str1 (28), CN str2 (12), CO ib1 (12) \\
\hline 1252 & 108 & $\mathrm{H}^{\alpha} \mathrm{b} 2(27), \mathrm{CN}$ str2(16), CN str1(14) \\
\hline 1170 & 1 & M2 rock1 (58) \\
\hline 1146 & 75 & $\mathrm{NC}^{\alpha} \operatorname{str}(41), \mathrm{C}^{\beta}$ rock2 (20) \\
\hline 1135 & 1 & M2 rock2(88) \\
\hline 1126 & 7 & $\mathrm{C}^{\alpha} \mathrm{C}^{\beta} \operatorname{str}(37), \mathrm{C}^{\beta} \operatorname{rock} 1(13)$ \\
\hline 1089 & 9 & $\mathrm{C}^{\beta}$ rock1 (24), NM str $(11)$ \\
\hline 1064 & 15 & $\mathrm{M} 1 \operatorname{rock2}(70), \mathrm{CO}$ ob1 (15) \\
\hline 1043 & 8 & $\mathrm{NM} \operatorname{str}(25), \mathrm{C}^{\beta}$ rock2(22), M1 rock1(19) \\
\hline 1001 & 12 & $\mathrm{M} 1$ rock $1(34), \mathrm{C}^{\alpha} \mathrm{C}^{\beta} \operatorname{str}(17), \mathrm{NM} \operatorname{str}(16)$ \\
\hline 943 & 7 & $\mathrm{MC} \operatorname{str}(32)$ \\
\hline 897 & 1 & $\mathrm{C}^{\beta} \operatorname{rock} 1(18), \mathrm{NC}^{\alpha} \operatorname{str}(14), \mathrm{CN} \operatorname{str} 1(12), \mathrm{CNC}^{\alpha} \operatorname{def}(11)$ \\
\hline 849 & 7 & $\mathrm{C}^{\alpha} \mathrm{C} \operatorname{str}(16), \mathrm{CN} \operatorname{str} 2(11), \mathrm{M} 2 \operatorname{rock} 1(11)$ \\
\hline 764 & 32 & $\mathrm{CO}$ ob2 (42), $\mathrm{CO}$ ib2(13) \\
\hline 672 & 164 & $\mathrm{NH}$ ob2 (42), $\mathrm{NH}$ ob1 (35), $\mathrm{CN}$ tor $2(20), \mathrm{CN}$ tor $1(17), \mathrm{NC}^{\alpha}$ tor (13) \\
\hline 657 & 1 & $\mathrm{CO}$ ob2(21), $\mathrm{CNC}^{\alpha} \operatorname{def}(14), \mathrm{MC} \operatorname{str}(14), \mathrm{CO} \mathrm{ib2}(12), \mathrm{CO} \mathrm{ib1}(11)$ \\
\hline
\end{tabular}


TABLE 4 (continued)

\begin{tabular}{|c|c|c|}
\hline$\nu\left(\mathrm{cm}^{-1}\right)$ & $\begin{array}{l}I^{\mathrm{a}} \\
\left(\mathrm{km} \mathrm{mol}^{-1}\right)\end{array}$ & Potential-energy distribution $>10 \%$ \\
\hline 628 & 16 & CO ob1 (74), M1 rock2(19) \\
\hline 571 & 81 & $\mathrm{CO}$ ib1 (30), CN tor2(14), $\mathrm{NH}$ ob2(11) \\
\hline 558 & 113 & $\begin{array}{l}\mathrm{NH} \text { ob2 (48), } \mathrm{NH} \text { ob1 }(38), \mathrm{CN} \text { tor } 1(23), \mathrm{CN} \text { tor } 2(21), \mathrm{NC}^{\alpha} \text { tor }(14) \text {, } \\
\mathrm{CO} \text { ib1(13) }\end{array}$ \\
\hline 480 & 25 & $\mathrm{C}^{\beta} \mathrm{b} 1(26), \mathrm{C}^{\alpha} \mathrm{CN} \operatorname{def}(22), \mathrm{CN}$ tor $1(11)$ \\
\hline 427 & 7 & $\mathrm{NC}^{\alpha} \mathrm{C} \operatorname{def}(38), \mathrm{MCN} \operatorname{def}(33), \mathrm{C}^{\beta} \mathrm{b} 2(16)$ \\
\hline 355 & 15 & CO ib2(21), MCN def(17), CNM def(15) \\
\hline 340 & 6 & $\mathrm{C}^{\beta} \mathrm{b} 2(38)$ \\
\hline 292 & 5 & CNM $\operatorname{def}(30), C^{\beta} b 1(13)$ \\
\hline 261 & 0 & $\mathrm{C}^{\alpha} \mathrm{C}^{\beta}$ tor $(88)$ \\
\hline 215 & 6 & $\mathrm{CNC}^{\alpha} \operatorname{def}(29), \mathrm{CN}$ tor2(23), NH ob2(12), MCN $\operatorname{def}(11)$ \\
\hline 196 & 2 & $\mathrm{C}^{\alpha} \mathrm{CN} \operatorname{def}(33), \mathrm{CNM} \operatorname{def}(17), \mathrm{C}^{\beta} \mathrm{b2}(12)$ \\
\hline 180 & 0 & $\mathrm{MC}$ tor $(100)$ \\
\hline 167 & 4 & $\mathrm{NM}$ tor $(32), \mathrm{CNC}^{\alpha} \operatorname{def}(20), \mathrm{NC}^{\alpha} \mathrm{C} \operatorname{def}(18), \mathrm{CN}$ tor2(12) \\
\hline 150 & 1 & NM tor $(96), \mathrm{NH}$ ob2(16) \\
\hline 108 & 9 & $\mathrm{CN}$ tor $1(41), \mathrm{NC}^{\alpha}$ tor $(14), \mathrm{C}^{\beta}$ b1 (11) \\
\hline 94 & 6 & $\mathrm{C}^{\alpha} \mathrm{C}$ tor $(123), \mathrm{CN}$ tor2 $(16)$ \\
\hline 81 & 6 & $\mathrm{NC}^{\alpha}$ tor $(85), \mathrm{NH}$ ob1 $(15), \mathrm{C}^{\alpha} \mathrm{C}$ tor $(10)$ \\
\hline
\end{tabular}

\begin{tabular}{|c|c|c|}
\hline$\alpha_{\mathrm{R}}$ & & \\
\hline 3467 & 33 & NH str2 (99) \\
\hline 3403 & 15 & NH str1(99) \\
\hline 3024 & 2 & MH1 str (86) \\
\hline 3014 & 1 & $\mathrm{CH} 14 \operatorname{str}(75), \mathrm{C}^{\alpha} \mathrm{H}^{\alpha} \operatorname{str}(12)$ \\
\hline 2998 & 1 & MH21 $\operatorname{str}(35), C^{\alpha} H^{\alpha} \operatorname{str}(32)$, MH22 str (21) \\
\hline 2998 & 7 & $\mathrm{C}^{\alpha} \mathrm{H}^{\alpha} \operatorname{str}(38), \mathrm{MH} 21 \operatorname{str}(28), \mathrm{MH} 22 \operatorname{str}(18)$ \\
\hline 2970 & 14 & MH20 str(49), MH22 str(34), MH21 str(19) \\
\hline 2968 & 1 & MH3 $\operatorname{str}(50), \mathrm{MH} 2 \operatorname{str}(48)$ \\
\hline 2960 & 18 & $\mathrm{CH} 15 \operatorname{str}(46), \mathrm{CH} 16 \operatorname{str}(31), \mathrm{C}^{\alpha} \mathrm{H}^{\alpha} \operatorname{str}(18)$ \\
\hline 2903 & 0 & $\mathrm{MH} 2 \operatorname{str}(45), \mathrm{MH} 3 \mathrm{str}(40), \mathrm{MH} 1 \mathrm{str}(14)$ \\
\hline 2899 & 35 & MH20 str(51), MH22 $\operatorname{str}(29)$, MH21 str(19) \\
\hline 2893 & 12 & $\mathrm{CH} 16 \operatorname{str}(56), \mathrm{CH} 15 \operatorname{str}(30), \mathrm{CH} 14 \operatorname{str}(13)$ \\
\hline 1726 & 236 & CO str $1(84)$ \\
\hline 1679 & 236 & CO str2(84) \\
\hline 1552 & 368 & NH ib2 (57), CN str2(33) \\
\hline 1526 & 320 & NH ibl(74), CN str1(20) \\
\hline 1477 & 3 & M2 ab1 (91) \\
\hline 1465 & 1 & $\mathrm{C}^{\beta} \mathrm{ab} 2(90)$ \\
\hline 1461 & 18 & $\mathrm{C}^{\beta}$ abl (93) \\
\hline 1455 & 10 & M2 ab2 (94) \\
\hline 1445 & 11 & M1 ab2 (93) \\
\hline 1435 & 24 & M1 ab1 (91) \\
\hline 1406 & 3 & M2 sb(105) \\
\hline 1371 & 27 & $\mathrm{M} 1 \mathrm{sb}(89), \mathrm{C}^{\beta} \mathrm{sb}(12)$ \\
\hline 1371 & 15 & $\mathrm{C}^{\beta} \mathrm{sb}(92), \mathrm{M} 1 \mathrm{sb}(12)$ \\
\hline 1322 & 6 & $\mathrm{H}^{\alpha} \mathrm{b} 2(89)$ \\
\hline
\end{tabular}


TABLE 4 (continued)

\begin{tabular}{|c|c|c|}
\hline$\nu\left(\mathrm{cm}^{-1}\right)$ & $\begin{array}{l}I^{\mathrm{a}} \\
\left(\mathrm{km} \mathrm{mol}^{-1}\right)\end{array}$ & Potential-energy distribution $>10 \%$ \\
\hline 1292 & 88 & $\mathrm{CN} \operatorname{str} 2(29), \mathrm{C}^{\alpha} \mathrm{C} \operatorname{str}(22), \mathrm{NH}$ ib2(22), $\mathrm{CO}$ ib2(12) \\
\hline 1277 & 119 & $\mathrm{H}^{\alpha} \mathrm{b} 1(44), \mathrm{CN} \operatorname{str} 1(19), \mathrm{CO} \mathrm{ib1}(10)$ \\
\hline 1253 & 99 & $\mathrm{H}^{\alpha}$ b1 (33), CN str1(24), NH ib1(11) \\
\hline 1166 & 7 & M2 rock1 (62) \\
\hline 1162 & 49 & $\mathrm{NC}^{\alpha} \operatorname{str}(35), \mathrm{C}^{\beta}$ rock2(19) \\
\hline 1125 & 6 & $\mathrm{M} 2$ rock2(86) \\
\hline 1108 & 7 & $\mathrm{C}^{\beta} \operatorname{rock} 1(31), \mathrm{C}^{\alpha} \mathrm{C}^{\beta} \operatorname{str}(30)$ \\
\hline 1097 & 12 & $\mathrm{NM} \operatorname{str}(36), \mathrm{C}^{\alpha} \mathrm{C} \operatorname{str}(11)$ \\
\hline 1063 & 13 & $\mathrm{M} 1 \operatorname{rock} 2(71), \mathrm{CO}$ ob1 (15) \\
\hline 1041 & 10 & $\mathrm{C}^{\beta}$ rock2 (28), $\mathrm{M} 1$ rock1 (23), $\mathrm{NM} \operatorname{str}(17)$ \\
\hline 996 & 10 & $\mathrm{M} 1 \operatorname{rock}(30), \mathrm{C}^{\alpha} \mathrm{C}^{\beta} \operatorname{str}(12), \mathrm{NM} \operatorname{str}(11)$ \\
\hline 931 & 4 & $M C \operatorname{str}(30), C^{\alpha} C^{\beta} \operatorname{str}(24)$ \\
\hline 899 & 2 & CN str1 (17), $\mathrm{C}^{\beta}$ rock1 (15), $\mathrm{C}^{\beta}$ rock2 (15), $\mathrm{CNC}^{\alpha} \operatorname{def}(13), \mathrm{NC}^{\alpha} \operatorname{str}(10)$ \\
\hline 844 & 8 & $\mathrm{C}^{\alpha} \mathrm{C} \operatorname{str}(16), \mathrm{CN} \operatorname{str} 2(12), \mathrm{CO}$ ib2 (12), CNM def(12), M2 rock1 (12) \\
\hline 758 & 22 & $\mathrm{CO}$ ob2 (56) \\
\hline 657 & 152 & $\mathrm{NH}$ ob2(28), $\mathrm{NH}$ ob1 (24), $\mathrm{CN}$ tor2(11) \\
\hline 651 & 21 & $\mathrm{CO}$ ob1 (42), M1 rock2(12), CN tor2(12) \\
\hline 637 & 58 & CO ib1 (19), MC str (17), NH ob1 (16) \\
\hline 592 & 6 & $\mathrm{CO}$ obl (34), CN tor $2(11)$ \\
\hline 547 & 82 & $\mathrm{NH}$ ob1 $(61), \mathrm{CN}$ tort $(47), \mathrm{NH}$ ob2 (36), $\mathrm{NC}^{\alpha}$ tor $(28), \mathrm{CN}$ tor2(18) \\
\hline 516 & 37 & $\mathrm{CO} \mathrm{ib} 1(22), \mathrm{MCN} \operatorname{def}(20), \mathrm{CO} \mathrm{ib2}(12), \mathrm{C}^{\alpha} \mathrm{CN} \operatorname{def}(10)$ \\
\hline 393 & 13 & $\mathrm{NC}^{\alpha} \mathrm{C} \operatorname{def}(27), \mathrm{C}^{\beta} \mathrm{bl}(25)$ \\
\hline 353 & 14 & MCN def(33), CO ib2(23) \\
\hline 333 & 8 & CNM def $(19), C^{\beta} b 1(12), C^{\beta}$ b2 (12), $C^{\alpha} C^{\beta}$ tor (12) \\
\hline 298 & 7 & $\mathrm{C}^{\alpha} \mathrm{C}^{\beta}$ tor $(50), \mathrm{CNM} \operatorname{def}(16)$ \\
\hline 276 & 4 & $\mathrm{C}^{\beta} \mathrm{b} 2(36), \mathrm{C}^{\alpha} \mathrm{C}^{\beta}$ tor $(33)$ \\
\hline 219 & 6 & $\mathrm{C}^{\alpha} \mathrm{CN} \operatorname{def}(27), \mathrm{CNM} \operatorname{def}(16)$ \\
\hline 203 & 1 & $\mathrm{C}^{\alpha} \mathrm{CN}$ def(14), $\mathrm{C}^{\alpha} \mathrm{C}$ tor (13), CN tor2(12), NH ob2(11) \\
\hline 183 & 0 & $\mathrm{MC} \operatorname{tor}(100)$ \\
\hline 165 & 2 & $\mathrm{CNC}^{\alpha} \operatorname{def}(30), \mathrm{NC}^{\alpha} \mathrm{C} \operatorname{def}(21), \mathrm{NM}$ tor $(14)$ \\
\hline 151 & 0 & NM tor (101) \\
\hline 110 & 6 & $\mathrm{CN}$ tor $1(46)$ \\
\hline 88 & 4 & $\mathrm{C}^{\alpha} \mathrm{C}$ tor $(63), \mathrm{CN}$ tor2(24), $\mathrm{CO}$ ob2(10) \\
\hline 78 & 6 & $\mathrm{NC}^{\alpha}$ tor $(137), \mathrm{C}^{\alpha} \mathrm{C}$ tor $(115), \mathrm{CO}$ ob2(14) \\
\hline \multicolumn{3}{|l|}{$\alpha_{\mathrm{L}}$} \\
\hline 3461 & 16 & NH str2(98) \\
\hline 3413 & 12 & NH str1 (98) \\
\hline 3030 & 1 & $\mathrm{CH} 16 \operatorname{str}(75), \mathrm{CH} 14 \operatorname{str}(19)$ \\
\hline 3023 & 2 & MH1 str (85) \\
\hline 3001 & 3 & $\mathrm{CH} 14 \operatorname{str}(63), \mathrm{CH} 15 \operatorname{str}(30)$ \\
\hline 2990 & 8 & MH22 str(62), MH20 str(34) \\
\hline 2973 & 15 & MH21 str (57), MH20 str(38) \\
\hline 2967 & 4 & MH2 $\operatorname{str}(52)$, MH3 $\operatorname{str}(49)$ \\
\hline
\end{tabular}


TABLE 4 (continued)

\begin{tabular}{|c|c|c|}
\hline$\nu\left(\mathrm{cm}^{-1}\right)$ & $\begin{array}{l}I^{\mathrm{a}} \\
(\mathrm{km} \mathrm{mol}\end{array}$ & Potential-energy distribution $>10 \%$ \\
\hline 2936 & 6 & $\mathrm{C}^{\alpha} \mathrm{H}^{\alpha} \operatorname{str}(95)$ \\
\hline 2911 & 22 & $\mathrm{CH} 15 \operatorname{str}(67), \mathrm{CH} 14 \operatorname{str}(18), \mathrm{CH} 16 \operatorname{str}(13)$ \\
\hline 2902 & 1 & MH3 $\operatorname{str}(44), \mathrm{MH} 2 \operatorname{str}(41), \mathrm{MH} 1 \mathrm{str}(14)$ \\
\hline 2900 & 34 & MH21 $\operatorname{str}(39)$, MH22 str(32), MH20 str(28) \\
\hline 1724 & 252 & $\mathrm{CO} \operatorname{str} 1(79)$ \\
\hline 1703 & 181 & $\mathrm{CO} \operatorname{str} 2(83)$ \\
\hline 1545 & 318 & NH ib2(61), CN str2(28) \\
\hline 1536 & 250 & NH ib1 (68), CN str1(19) \\
\hline 1478 & 2 & M2 ab1 (92) \\
\hline 1476 & 12 & $C^{\beta}$ ab2(89) \\
\hline 1455 & 5 & M2 ab2(94) \\
\hline 1451 & 14 & $\mathrm{C}^{\beta} \mathrm{ab1}(90)$ \\
\hline 1446 & 11 & M1 ab2(93) \\
\hline 1434 & 24 & M1 ab1 (91) \\
\hline 1409 & 2 & $\mathrm{M} 2 \mathrm{sb}(104)$ \\
\hline 1374 & 16 & $\mathrm{C}^{\beta} \mathrm{sb}(104)$ \\
\hline 1371 & 31 & M1 sb(101) \\
\hline 1314 & 52 & $\mathrm{H}^{\alpha} \mathrm{b} 2(62), \mathrm{C}^{\alpha} \mathrm{C} \operatorname{str}(12), \mathrm{NC}^{\alpha} \operatorname{str}(11)$ \\
\hline 1311 & 29 & $\mathrm{H}^{\alpha} \mathrm{b} 1(57), \mathrm{C}^{\alpha} \mathrm{C} \operatorname{str}(12)$ \\
\hline 1265 & 183 & CN str1 (39), CO ibl (17), MC str(14), NH ibl(13) \\
\hline 1247 & 81 & CN str2(26), $\mathrm{H}^{\alpha} \mathrm{b} 2(22), \mathrm{H}^{\alpha} \mathrm{b} 1(13), \mathrm{NM} \operatorname{str}(11)$ \\
\hline 1171 & 2 & $\mathrm{M} 2 \operatorname{rock} 1(55)$ \\
\hline 1145 & 29 & $\mathrm{NC}^{\alpha} \operatorname{str}(27), \mathrm{C}^{\beta}$ rock2(27) \\
\hline 1138 & 4 & $\mathrm{C}^{\alpha} \mathrm{C}^{\beta} \operatorname{str}(30), \mathrm{C}^{\beta}$ rockl (19) \\
\hline 1135 & 3 & $\mathrm{M} 2 \operatorname{rock} 2(84)$ \\
\hline 1077 & 5 & $\mathrm{NM} \operatorname{str}(20), \mathrm{C}^{\beta} \operatorname{rock} 1(20), \mathrm{H}^{\alpha} \mathrm{b} 1(12), \mathrm{C}^{\alpha} \mathrm{C} \operatorname{str}(10)$ \\
\hline 1063 & 19 & M1 rock2(68), CO ob1(15) \\
\hline 1047 & 5 & $\mathrm{C}^{\beta}$ rock2(30), M1 rock1(18), NM str(17) \\
\hline 997 & 18 & $\mathrm{M} 1$ rock1 (31), NM $\operatorname{str}(18), \mathrm{C}^{\alpha} \mathrm{C}^{\beta} \operatorname{str}(17), \mathrm{MC} \operatorname{str}(11)$ \\
\hline 924 & 4 & $\mathrm{MC} \operatorname{str}(20), \mathrm{C}^{\alpha} \mathrm{C}^{\beta} \operatorname{str}(17), \mathrm{C}^{\beta} \operatorname{rock} 1(12), \mathrm{C}^{\beta} \operatorname{rock} 2(11)$ \\
\hline 891 & 1 & $\mathrm{CN} \operatorname{str1}(19), \mathrm{CNC}^{\alpha} \operatorname{def}(17)$ \\
\hline 827 & 13 & $\mathrm{CO}$ ib2 (14), CNM def(12), M2 rock1 (12), $\mathrm{C}^{\alpha} \mathrm{C} \operatorname{str}(11)$ \\
\hline 772 & 34 & $\mathrm{CO}$ ob2 (53), $\mathrm{NC}^{\alpha} \mathrm{C} \operatorname{def}(15)$ \\
\hline 659 & 8 & $\mathrm{MC} \operatorname{str}(23), \mathrm{CO} \mathrm{ib} 1(19), \mathrm{CO} \mathrm{ib} 2(10), \mathrm{C}^{\beta} \mathrm{b} 2(10)$ \\
\hline 642 & 27 & CO ob1 (66), M1 rock2(19) \\
\hline 585 & 106 & $\mathrm{CN}$ tor2 $(44), \mathrm{NH}$ ob2 $(40)$ \\
\hline 573 & 45 & $\mathrm{NH}$ ob2 (40), CN tor2(13), $\mathrm{C}^{\alpha} \mathrm{C} \operatorname{str}(12), \mathrm{NH}$ ob1 (12), $\mathrm{CO}$ ob2(10) \\
\hline 546 & 170 & NH ob1 (113), CN tor $1(67), \mathrm{NC}^{\alpha}$ tor $(65)$ \\
\hline 487 & 15 & $\mathrm{C}^{\beta} \mathrm{b} 1(30), \mathrm{C}^{\alpha} \mathrm{CN} \operatorname{def}(21)$ \\
\hline 457 & 27 & $\mathrm{CO} \mathrm{ib} 1(40), \mathrm{NC}^{\alpha} \mathrm{C} \operatorname{def}(26)$ \\
\hline 349 & 12 & CNM $\operatorname{def}(17), C^{\beta} b 1(15), C^{\beta} b 2(15), M C N \operatorname{def}(14)$ \\
\hline 338 & 11 & $\mathrm{C}^{\beta} \mathrm{b} 2(29), \mathrm{NC}^{\alpha} \mathrm{C} \operatorname{def}(13), \mathrm{CO} \mathrm{ib2}(12), \mathrm{MCN} \operatorname{def}(12)$ \\
\hline 301 & 9 & $\mathrm{MCN} \operatorname{def}(32), \mathrm{CNM} \operatorname{def}(25), \mathrm{C}^{\beta} \mathrm{b} 2(14), \mathrm{C}^{\beta} \mathrm{b} 1(12)$ \\
\hline 268 & 1 & $\mathrm{C}^{\alpha} \mathrm{C}^{\beta}$ tor $(93)$ \\
\hline 205 & 0 & $\mathrm{CNC}^{\alpha} \operatorname{def}(23), \mathrm{CN}$ tor2 (17), $\mathrm{C}^{\alpha} \mathrm{C}$ tor (16), $\mathrm{NH}$ ob2 (14), $\mathrm{C}^{\alpha} \mathrm{CN} \operatorname{def}(11)$ \\
\hline
\end{tabular}


TABLE 4 (continued)

\begin{tabular}{|c|c|c|}
\hline$\nu\left(\mathrm{cm}^{-1}\right)$ & $\begin{array}{l}I^{\mathrm{a}} \\
\left(\mathrm{km} \mathrm{mol}^{-1}\right)\end{array}$ & Potential-energy distribution $>10 \%$ \\
\hline 199 & 2 & $\mathrm{C}^{\alpha} \mathrm{CN} \operatorname{def}(34), \mathrm{CNM} \operatorname{def}(21)$ \\
\hline 186 & 1 & $\mathrm{MC}$ tor $(89)$ \\
\hline 177 & 3 & $\mathrm{NC}^{\alpha}$ tor $(22), \mathrm{CNC}^{\alpha} \operatorname{def}(20), \mathrm{NM}$ tor (17), $\mathrm{MC}$ tor (16) \\
\hline 154 & 1 & NM tor (105), NH ob2(13) \\
\hline 113 & 11 & $\mathrm{CN}$ tor $1(36), \mathrm{NC}^{\alpha}$ tor $(18)$ \\
\hline 107 & 4 & $\mathrm{C}^{\alpha} \mathrm{C}$ tor $(116), \mathrm{NC}^{\alpha}$ tor $(36), \mathrm{CN}$ tor $2(12)$ \\
\hline 88 & 2 & $\mathrm{NC}^{\alpha}$ tor $(42), \mathrm{CN}$ tor $1(14)$ \\
\hline
\end{tabular}

$\alpha^{\prime}$

\begin{tabular}{|c|c|c|}
\hline 3474 & 49 & NH str2(99) \\
\hline 3418 & 24 & NH str1(100) \\
\hline 3024 & 2 & MH1 str(85) \\
\hline 3019 & 1 & $\mathrm{C}^{\alpha} \mathrm{H}^{\alpha} \operatorname{str}(51), \mathrm{CH} 15 \operatorname{str}(34), \mathrm{CH} 16 \operatorname{str}(12)$ \\
\hline 3004 & 3 & $\mathrm{CH} 15 \operatorname{str}(49), \mathrm{C}^{\alpha} \mathrm{H}^{\alpha} \operatorname{str}(32), \mathrm{CH} 14 \operatorname{str}(18)$ \\
\hline 2996 & 6 & MH22 str(65), MH20 str(26) \\
\hline 2977 & 14 & MH21 str(53), MH20 str(45) \\
\hline 2969 & 3 & MH3 $\operatorname{str}(31), \mathrm{MH} 2 \operatorname{str}(30), \mathrm{CH} 14 \operatorname{str}(19), \mathrm{CH} 16 \operatorname{str}(18)$ \\
\hline 2967 & 7 & CH14 str (25), CH16 str(24), MH3 str(22), MH2 $\operatorname{str}(19), \mathrm{C}^{\alpha} \mathrm{H}^{\alpha} \operatorname{str}(11)$ \\
\hline 2905 & 30 & MH21 $\operatorname{str}(40)$, MH22 $\operatorname{str}(30)$, MH20 str (29) \\
\hline 2902 & 1 & MH2 $\operatorname{str}(44)$, MH3 $\operatorname{str}(40)$, MH1 $\operatorname{str}(14)$ \\
\hline 2900 & 13 & CH16 str (46), CH14 str(37), CH15 str(15) \\
\hline 1720 & 247 & CO str2(83) \\
\hline 1710 & 207 & CO str1 (82) \\
\hline 1540 & 277 & NH ib2(65), CN str2(25) \\
\hline 1529 & 421 & NH ib1 (68), CN str1 (22) \\
\hline 1477 & 2 & M2 ab1 (91) \\
\hline 1470 & 18 & $\mathrm{C}^{\beta} \mathrm{abl}(90)$ \\
\hline 1454 & 8 & $\mathrm{C}^{\beta} \mathrm{ab2}(87)$ \\
\hline 1454 & 7 & M2 ab2(92) \\
\hline 1446 & 11 & M1 ab2(94) \\
\hline 1435 & 29 & M1 ab1 (91) \\
\hline 1412 & 0 & M2 sb(104) \\
\hline 1374 & 9 & $\mathrm{C}^{\beta} \mathrm{sb}(100)$ \\
\hline 1371 & 29 & M1 sb(99) \\
\hline 1326 & 9 & $\mathrm{H}^{\alpha} \mathrm{b} 1(55), \mathrm{H}^{\alpha} \mathrm{b} 2(28)$ \\
\hline 1295 & 158 & $\mathrm{C}^{\alpha} \mathrm{C} \operatorname{str}(28), \mathrm{CN}$ str2(27), NH ib2(12), CO ib2(11) \\
\hline 1291 & 51 & $\mathrm{CN} \operatorname{str} 1(21), \mathrm{H}^{\alpha} \mathrm{b} 2(18), \mathrm{H}^{\alpha} \mathrm{b} 1(16)$ \\
\hline 1260 & 96 & $\mathrm{H}^{\alpha} \mathrm{b} 2(37), \mathrm{CN} \operatorname{str1}(19), \mathrm{NH}$ ib1(12) \\
\hline 1169 & 4 & $\mathrm{M} 2 \operatorname{rock} 1(58), \mathrm{NM} \operatorname{str}(11)$ \\
\hline 1137 & 21 & $\mathrm{NC}^{\alpha} \operatorname{str}(32), \mathrm{M} 2$ rock2(28) \\
\hline 1136 & 8 & $\mathrm{M} 2 \operatorname{rock} 2(61), \mathrm{NC}^{\alpha} \operatorname{str}(15)$ \\
\hline 1110 & 16 & NM str (42) \\
\hline 1099 & 8 & $\mathrm{C}^{\alpha} \mathrm{C}^{\beta} \operatorname{str}(35), \mathrm{C}^{\beta} \operatorname{rock} 1(31)$ \\
\hline 1063 & 17 & $\mathrm{M} 1 \operatorname{rock} 2(71), \mathrm{CO}$ ob1 (15) \\
\hline 1018 & 2 & $\mathrm{M} 1$ rock $1(39), \mathrm{C}^{\beta}$ rock2(20) \\
\hline 979 & 27 & $\mathrm{C}^{\beta}$ rock2 (23), M1 rock1 (16), NM str(14) \\
\hline
\end{tabular}


TABLE 4 (continued)

\begin{tabular}{|c|c|c|}
\hline$\nu\left(\mathrm{cm}^{-1}\right)$ & $\begin{array}{l}I^{\mathrm{a}} \\
\left(\mathrm{km} \mathrm{mol}^{-1}\right)^{\mathrm{I}}\end{array}$ & Potential-energy distribution $>10 \%$ \\
\hline 947 & 8 & $\mathrm{MC} \operatorname{str}(33), \mathrm{CNC}^{\alpha} \operatorname{def}(10)$ \\
\hline 899 & 4 & $\mathrm{C}^{\alpha} \mathrm{C}^{\beta} \operatorname{str}(21), \mathrm{C}^{\beta} \operatorname{rock} 1(16), \mathrm{NC}^{\alpha} \operatorname{str}(15)$ \\
\hline 840 & 6 & $\mathrm{CN}$ str2(17), M2 rock1(13), CNM def(12), $\mathrm{C}^{\alpha} \mathrm{C} \operatorname{str}(12), \mathrm{CO} \mathrm{ib2}(11)$ \\
\hline 765 & 39 & $\mathrm{CO}$ ob2 $(71)$ \\
\hline 681 & 8 & $\mathrm{CO}$ ib2(19), MC str(17) \\
\hline 640 & 30 & $\mathrm{CO}$ ob1 (63), $\mathrm{M} 1$ rock2(17) \\
\hline 605 & 98 & $\begin{array}{l}\mathrm{NH} \text { ob2 }(47), \mathrm{NH} \text { ob1 }(45), \mathrm{CN} \text { tor2 }(29), \mathrm{CN} \text { tor } 1(21), \mathrm{NC}^{\alpha} \text { tor }(16) \text {, } \\
\mathrm{CO} \text { ob1(11) }\end{array}$ \\
\hline 594 & 10 & $\mathrm{CO}$ ib1 (30), NC ${ }^{\alpha} \mathrm{C} \operatorname{def}(17), \mathrm{C}^{\alpha} \mathrm{CN} \operatorname{def}(11)$ \\
\hline 524 & 31 & CO ib1 (20), NC ${ }^{\alpha} \mathrm{C} \operatorname{def}(17), \mathrm{CO} i b 2(16), \mathrm{MCN} \operatorname{def}(11)$ \\
\hline 504 & 163 & $\mathrm{NH}$ ob1 (71), NH ob2(55), CN tor1(53), $\mathrm{NC}^{\alpha}$ tor $(43), \mathrm{CN}$ tor2(38) \\
\hline 375 & 21 & MCN $\operatorname{def}(48), \mathrm{C}^{\beta} \mathrm{b} 1(13), \mathrm{CO} \mathrm{ib} 1(11)$ \\
\hline 366 & 11 & $\mathrm{C}^{\beta} \mathrm{b} 2(24), \mathrm{CO}$ ib2(22), $\mathrm{C}^{\beta} \mathrm{b} 1(13), \mathrm{NC}^{\alpha} \mathrm{C} \operatorname{def}(11)$ \\
\hline 305 & 8 & CNM $\operatorname{def}(41), \mathrm{C}^{\beta} \mathrm{b} 2(27)$ \\
\hline 287 & 0 & $\mathrm{C}^{\alpha} \mathrm{C}^{\beta}$ tor $(99)$ \\
\hline 264 & 5 & $\mathrm{C}^{\beta} \mathrm{b} 1(17), \mathrm{C}^{\beta}$ b2 (17), NH ob2(15), CN tor2(11) \\
\hline 218 & 7 & $\mathrm{CNC}^{\alpha} \operatorname{def}(35), \mathrm{C}^{\alpha} \mathrm{CN} \operatorname{def}(26), \mathrm{MCN} \operatorname{def}(13), \mathrm{CNM} \operatorname{def}(13)$ \\
\hline 191 & 2 & $\mathrm{CN}$ tor2 (17), $\mathrm{MC}$ tor $(10), \mathrm{C}^{\beta} \mathrm{b} 2(10)$ \\
\hline 179 & 0 & $\mathrm{MC}$ tor $(96)$ \\
\hline 161 & 1 & $\mathrm{NM}$ tor (117), NH ob2(12), M2 rock2(10) \\
\hline 148 & 7 & $\mathrm{C}^{\alpha} \mathrm{C}$ tor (54), $\mathrm{NH}$ ob1 (28), $\mathrm{CN}$ tor1(12), $\mathrm{C}^{\alpha} \mathrm{CN} \operatorname{def}(10)$ \\
\hline 120 & 5 & $\mathrm{CN}$ torl $(31), \mathrm{C}^{\beta}$ b1 $(24), \mathrm{C}^{\alpha} \mathrm{C}$ tor $(14)$ \\
\hline 110 & 8 & $\mathrm{NC}^{\alpha}$ tor $(57), \mathrm{CN}$ tor2(15) \\
\hline 85 & 4 & $\mathrm{C}^{\alpha} \mathrm{C}$ tor $(83), \mathrm{NC}^{\alpha}$ tor $(43), \mathrm{CN}$ tor $2(18)$ \\
\hline
\end{tabular}

${ }^{\mathrm{a}}$ Infrared intensity.

in the open structures to increase as the $\mathrm{OCNH}$ dihedral angle deviates from planarity $\left(180^{\circ}\right)$, whereas those in the $\mathrm{C}_{5}$ and $\mathrm{C}_{7}$ structures decrease. Figure $2(\mathrm{~d})$ shows that, although the $\mathrm{CO}$ stretch force constants in the $\beta_{2}$ and $\alpha$ structures do not correlate well with $\omega$ (the $\mathrm{CC}(0) \mathrm{NC}$ dihedral angle), those in the hydrogen-bonded conformers decrease almost linearly as $\omega$ departs from $180^{\circ}$. Because the non-planarity of the $\mathrm{CONH}$ group reduces the electronic resonance in the $\mathrm{CO}$ and $\mathrm{CN}$ bonds, the increase in $\mathrm{f}(\mathrm{CO}$ str $)$ with non-planarity, as measured by the OCNH angle, in the open structures is not surprising. The lack of correlation with $\omega$ probably reflects the fact that this angle is not as direct a measure of the planarity of $\mathrm{CONH}$ when the out-of-plane angles at $\mathrm{C}$ and $\mathrm{N}$ are sizeable. That $\mathrm{f}(\mathrm{CO} \mathrm{str})$ in the $\mathrm{C}_{5}$ and $\mathrm{C}_{7}$ conformers decrease with non-planarity suggests that higher degrees of non-planarity are associated with stronger hydrogen bonds. Such significant variations in force constants with the peptide group planarity are important because the deviations from planarity seen in these Ala dipeptide conformers ( $\omega$ ranging from $174.5^{\circ}$ to $187.6^{\circ}$ [4]) are not unusual in actual peptide structures. A recent crystal-structure 

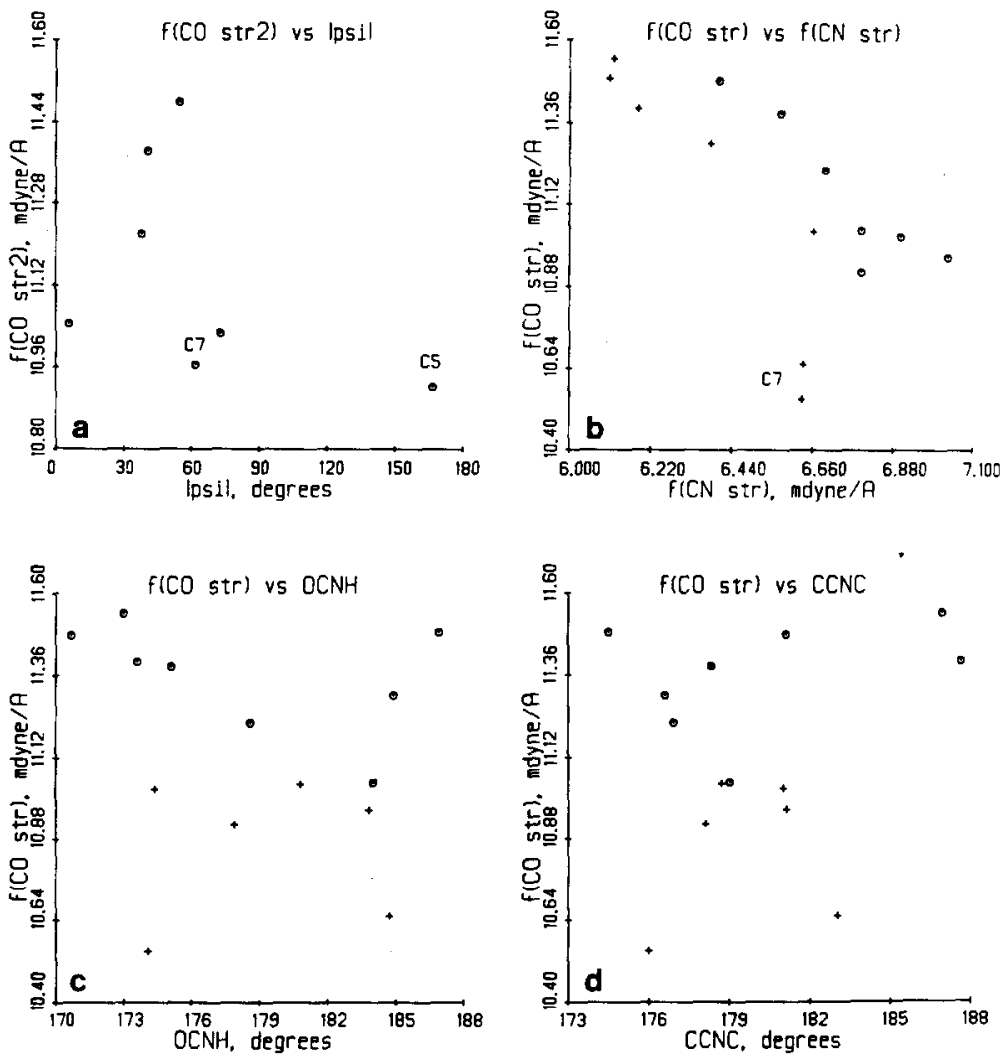

Fig. 2. Plots of the scaled $\mathrm{CO}$ stretch force constant: (a) versus $|\psi|$; (b) versus scaled $\mathrm{CN}$ stretch force constant; (c) versus OCNH dihedral angle; and (d) versus $\mathrm{CC}(\mathrm{O}) \mathrm{NC}$ dihedral angle. (b) $\times, f(C O$ str 1$)$ and $f(C N$ str 1$) ; O, f(C O$ str2 $)$ and $f(C N$ str2 $)$ (c) and $(d): \times, C_{5}$ and $\mathrm{C}_{7}$ conformers; $O$, open conformers.

survey [15] found values of $\omega$ in the range $157-201^{\circ}$, and in crystalline Ala dipeptide itself $\omega$ values of $171.9-191.6^{\circ}$ were derived in an X-ray study [16]. The $\mathrm{CO}$ and $\mathrm{NH}$ in-plane and out-of-plane bend (ib and ob) force constants do not vary significantly in the open conformations. Among the other diagonal and off-diagonal terms, as we concluded previously [1,2], it is primarily those involving the $\mathrm{C}^{\alpha}$ atom that vary appreciably with conformation. Also notable are the changes in the $\mathrm{C}^{\beta} \mathrm{H}$ stretches.

To summarize some of our results on the force fields of these Gly and Ala dipeptide structures, we again plot in Fig. 3 the scaled $\mathrm{NH}, \mathrm{CO}, \mathrm{CN}$ and $\mathrm{CH}$ stretch force constants against their respective equilibrium bond lengths. As before [2], we have fitted each set of points with a straight line, and the following least-squares slopes result (in units of mdyn $\AA^{-2}$ ): $-62,-75,-31$, and -35 , respectively. Except for the $\mathrm{CH}$ stretch slope, these are smaller than 

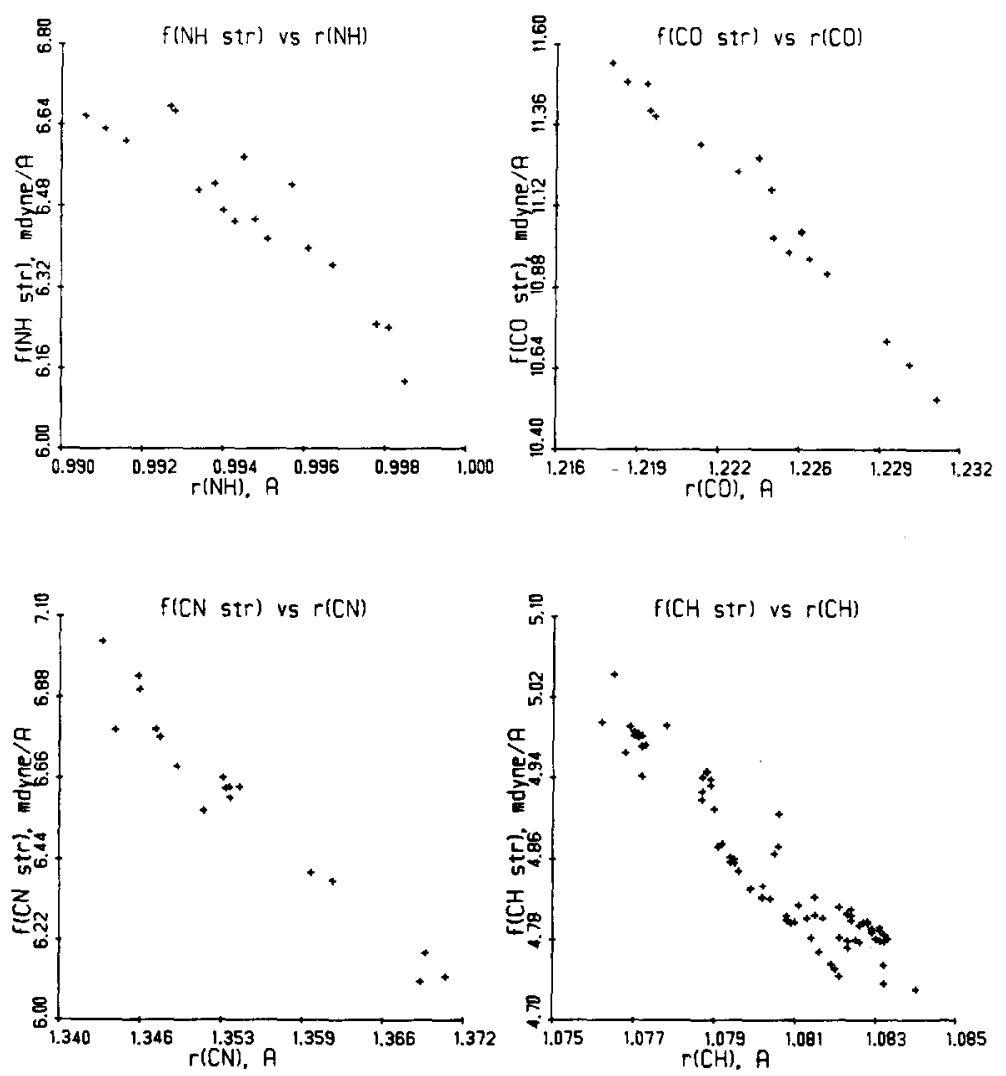

Fig. 3. Scaled $\mathrm{NH}, \mathrm{CO}, \mathrm{CN}$ and $\mathrm{CH}$ stretch diagonal force constants plotted against their respective equilibrium bond lengths.

obtained previously with much smaller sets of points. Even so, we continue to see close agreements of these slopes with the average diagonal cubic force constants, the values of which are $-63,-85,-48$, and -35 mdyn $\AA^{-2}$, respectively.

Turning to the normal modes, some of the amide modes in the seven Ala dipeptide conformations are summarized in Table 5 . In the $1200-1300 \mathrm{~cm}^{-1}$ region, the amide III mixes strongly, and to varying degrees, with the $\mathrm{H}^{\alpha}$ bends $\left(\mathrm{H}^{\alpha} \mathrm{b}\right)$. This mixing can be removed by looking at the "pure" amide III in $\mathrm{CD}^{\alpha}$-Ala dipeptide and the "pure" $\mathrm{H}^{\alpha} \mathrm{b}$ in the $N$-deuterated isotopomer; the resulting frequencies are given in Table 5 .

Because of their localized nature, the $\mathrm{NH}$ stretch frequencies reflect closely the differences in the force constants that were discussed above. Notable are the substantially lowered frequencies on hydrogen bonding, the systematically higher free $\mathrm{NH}$ str2 modes compared with the free $\mathrm{NH}$ str1, and the fact that both $\mathrm{NH}$ stretches show some sensitivity to conformation. 
TABLE 5

Some calculated frequencies (in $\mathrm{cm}^{-1}$ ) in normal and selectively deuterated Ala dipeptides

\begin{tabular}{|c|c|c|c|c|c|c|c|}
\hline Mode & $\mathrm{C}_{5}$ & $\mathrm{C}_{7}^{\mathrm{eq}}$ & $\mathrm{C}_{7}^{\mathrm{ax}}$ & $\beta_{2}$ & $\alpha_{\mathrm{R}}$ & $\alpha_{\mathrm{L}}$ & $\alpha^{\prime}$ \\
\hline NH str2 & 3471 & 3360 & 3334 & 3455 & 3467 & 3462 & 3474 \\
\hline NH str1 & 3390 & 3414 & 3446 & 3431 & 3403 & 3413 & 3418 \\
\hline Amide I (CO str2) & 1698 & 1704 & 1699 & 1694 & 1679 & 1703 & 1720 \\
\hline Amide I (CO str1) & 1668 & 1676 & 1682 & 1719 & 1726 & 1725 & 1710 \\
\hline Amide II (NH ib2) & 1557 & 1587 & 1594 & 1550 & 1552 & 1546 & 1540 \\
\hline Amide II (NH ib1) & 1525 & 1534 & 1551 & 1532 & 1526 & 1536 & 1529 \\
\hline Amide III (CN str2) /CD ${ }^{\alpha}$ & 1321 & 1322 & 1327 & 1295 & 1294 & 1292 & 1295 \\
\hline Amide III (CN str 1$) / \mathrm{CD}^{\alpha}$ & 1276 & 1286 & 1294 & 1262 & 1263 & 1262 & 1278 \\
\hline \multirow[t]{2}{*}{$\mathrm{H}^{\alpha}$ bend $/(\mathrm{ND})_{2}$} & 1315 & 1314 & 1317 & 1300 & 1320 & 1305 & 1324 \\
\hline & 1288 & 1305 & 1274 & 1296 & 1272 & 1283 & 1284 \\
\hline \multirow[t]{2}{*}{$\mathrm{C}^{\beta} \mathrm{H}_{3}$ asym str $/ \mathrm{CD}^{\alpha}$} & 3014 & 2997 & 3038 & 3010 & 3011 & 3028 & 3010 \\
\hline & 2979 & 2990 & 2977 & 2977 & 2968 & 3001 & 2974 \\
\hline $\mathrm{CH}^{\alpha}$ str $/\left(\mathrm{CD}_{3}\right)_{3}$ & 2955 & 2991 & 3000 & 2985 & 2993 & 2940 & 3007 \\
\hline
\end{tabular}

In the open conformers, the amide I modes consisting primarily of CO str1 are quite constant in frequency, whereas the $\mathrm{CO}$ str2 vibrations vary significantly, as would be expected because $\mathrm{CO}$ str 2 is adjacent to $\mathrm{C}^{\alpha}$. The much lower $\mathrm{CO}$ str 1 frequencies in $\mathrm{C}_{7}^{\text {eq }}$ and $\mathrm{C}_{7}^{\text {ax }}$ arise from the $\mathrm{C}_{7}$ hydrogen bonds; the amide I modes in $\mathrm{C}_{5}$ are the most delocalized [2] and, consequently, do not reflect clearly the $\mathrm{C}_{5}$ hydrogen bond. The amide II modes are seen to be hardly sensitive to conformation in the non-hydrogen bonded structures. Thus, in polypeptides the different amide II frequencies observed in different conformations [17] arise mainly from differences in hydrogen-bond strength and from transition dipole-dipole interaction $[17,18]$.

The amide III modes, when $\mathrm{C}^{\alpha}$ is deuterated, hardly vary at all in the open conformers (with the exception of $\alpha^{\prime}$ ), and are systematically higher in frequency in the hydrogen-bonded structures. On the other hand, on $N$-deuteration, the $\mathrm{H}^{\alpha} \mathrm{b}$ modes vary markedly across all conformers. Thus, in normal trans peptides the amide III is sensitive to hydrogen bonding through its $\mathrm{CN}$ str and $\mathrm{NH}$ ib components, and to conformation mainly through its $\mathrm{H}^{\alpha}$ b contributions.

Also listed in Table 5 are the $\mathrm{C}^{\beta} \mathrm{H}_{3}$ antisymmetric stretches (with $\mathrm{C}^{\alpha}$ deuterated to remove accidental mixings) and the $\mathrm{CH}^{\alpha}$ str (with $\mathrm{C}^{\beta}$ deuterated) to demonstrate that these modes are also affected by conformation, as we had 
TABLE 6

Dipole-moment derivatives $\partial \vec{\mu} / \partial S$ (in $\mathrm{D} \AA^{-1}$ or $\mathrm{D} \mathrm{rad}{ }^{-1}$ ) of Ala dipeptides referred to group axes"

\begin{tabular}{|c|c|c|c|c|c|}
\hline & $\partial \mu_{x} / \partial S$ & $\partial \mu_{y} / \partial S$ & $\partial \mu_{z} / \partial S$ & $|\partial \vec{\mu} / \partial S|$ & $\theta^{\mathbf{b}}$ \\
\hline \multicolumn{6}{|l|}{ C6 N7 O5 } \\
\hline \multirow{7}{*}{ NH str1 } & 0.90 & 0.43 & 0.04 & 1.00 & 26 \\
\hline & 0.32 & 0.40 & 0.03 & 0.51 & 51 \\
\hline & 0.67 & 0.75 & -0.09 & 1.01 & 48 \\
\hline & 0.51 & 0.64 & -0.16 & 0.83 & 51 \\
\hline & 0.29 & 0.21 & -0.21 & 0.41 & 35 \\
\hline & 0.25 & 0.20 & 0.18 & 0.36 & 39 \\
\hline & 0.41 & 0.22 & 0.29 & 0.54 & 28 \\
\hline \multirow[t]{7}{*}{ CO str1 } & 4.23 & 4.47 & -0.01 & 6.15 & 47 \\
\hline & 4.25 & 5.10 & -0.14 & 6.64 & 50 \\
\hline & 3.99 & 5.22 & 0.16 & 6.57 & 53 \\
\hline & 4.65 & 4.53 & -0.00 & 6.49 & 44 \\
\hline & 4.52 & 4.24 & 0.24 & 6.20 & 43 \\
\hline & 3.97 & 4.07 & -0.12 & 5.68 & 46 \\
\hline & 4.34 & 4.48 & -0.05 & 6.24 & 46 \\
\hline \multirow[t]{7}{*}{ CN str1 } & -4.70 & -0.20 & 0.11 & 4.71 & -178 \\
\hline & -4.07 & -0.22 & -0.05 & 4.08 & -177 \\
\hline & -3.74 & -0.12 & -0.09 & 3.74 & -178 \\
\hline & -4.72 & -0.06 & -0.12 & 4.72 & -179 \\
\hline & -4.30 & -0.02 & -0.40 & 4.32 & -180 \\
\hline & -3.41 & -0.22 & -0.02 & 3.41 & -176 \\
\hline & -4.47 & -0.24 & 0.03 & 4.48 & -177 \\
\hline \multirow[t]{7}{*}{$\mathrm{NC}^{\alpha}$ str } & 1.69 & -1.32 & -0.41 & 2.19 & -38 \\
\hline & 1.96 & -2.09 & 0.10 & 2.87 & -47 \\
\hline & 2.11 & -2.52 & 0.21 & 3.29 & -50 \\
\hline & 1.56 & -1.48 & -0.55 & 2.21 & -44 \\
\hline & 1.46 & -1.36 & -0.23 & 2.01 & -43 \\
\hline & 1.56 & -1.59 & 0.53 & 2.29 & -45 \\
\hline & 1.11 & -1.40 & -0.47 & 1.85 & -52 \\
\hline \multirow[t]{7}{*}{ MCN def } & -1.99 & 0.89 & 0.05 & 2.18 & 156 \\
\hline & -1.54 & 1.35 & -0.20 & 2.06 & 139 \\
\hline & -1.69 & 1.31 & 0.20 & 2.15 & 142 \\
\hline & -1.32 & 0.92 & -0.19 & 1.63 & 145 \\
\hline & -0.93 & 0.68 & -0.14 & 1.16 & 144 \\
\hline & -0.83 & 0.50 & 0.16 & 0.98 & 149 \\
\hline & -1.52 & 0.59 & -0.04 & 1.63 & 159 \\
\hline \multirow[t]{7}{*}{$\mathrm{CO} \mathrm{ibl}$} & -3.16 & 2.14 & 0.08 & 3.81 & 146 \\
\hline & -2.92 & 2.43 & -0.34 & 3.81 & 140 \\
\hline & -2.86 & 2.68 & 0.24 & 3.93 & 137 \\
\hline & -2.87 & 2.00 & -0.05 & 3.50 & 145 \\
\hline & -2.69 & 1.94 & -0.15 & 3.31 & 144 \\
\hline & -2.75 & 2.07 & 0.15 & 3.45 & 143 \\
\hline & -2.99 & 1.92 & 0.05 & 3.56 & 147 \\
\hline
\end{tabular}


TABLE 6 (continued)

\begin{tabular}{|c|c|c|c|c|c|}
\hline & $\partial \mu_{x} / \partial S$ & $\partial \mu_{y} / \partial S$ & $\partial \mu_{2} / \partial S$ & $|\partial \vec{\mu} / \partial S|$ & $\theta^{\mathrm{b}}$ \\
\hline \multirow[t]{7}{*}{$\mathrm{CNC}^{\alpha}$ def } & 4.20 & -1.38 & -0.06 & 4.42 & -18 \\
\hline & 2.84 & -3.08 & 0.23 & 4.20 & -47 \\
\hline & 2.79 & -3.44 & -0.16 & 4.43 & -51 \\
\hline & 1.83 & -1.98 & 0.12 & 2.70 & -47 \\
\hline & 1.03 & -1.30 & -0.17 & 1.67 & -52 \\
\hline & 0.96 & -1.00 & 0.05 & 1.39 & -46 \\
\hline & 2.40 & -1.05 & 0.19 & 2.63 & -24 \\
\hline \multirow[t]{7}{*}{ NH ib1 } & 0.91 & -0.37 & 0.14 & 0.99 & -22 \\
\hline & 0.53 & -0.26 & -0.01 & 0.59 & -26 \\
\hline & 0.50 & -0.11 & -0.05 & 0.52 & -13 \\
\hline & 0.42 & -0.24 & -0.30 & 0.57 & -30 \\
\hline & 0.46 & -0.24 & -0.34 & 0.62 & -27 \\
\hline & 0.43 & -0.11 & 0.33 & 0.55 & -14 \\
\hline & 0.68 & -0.25 & 0.24 & 0.76 & -20 \\
\hline \multirow[t]{7}{*}{ CO obl } & -0.09 & 0.01 & 0.80 & 0.81 & 174 \\
\hline & 0.20 & -0.02 & 1.52 & 1.54 & -7 \\
\hline & -0.05 & 0.05 & 1.63 & 1.63 & 133 \\
\hline & 0.03 & -0.09 & 1.16 & 1.16 & -72 \\
\hline & 0.11 & -0.05 & 1.11 & 1.12 & -23 \\
\hline & -0.11 & 0.15 & 1.16 & 1.17 & 126 \\
\hline & -0.43 & 0.28 & 1.21 & 1.31 & 147 \\
\hline \multirow[t]{7}{*}{ NH ob1 } & 0.01 & 0.04 & 2.27 & 2.27 & 74 \\
\hline & -0.26 & -0.13 & 1.03 & 1.07 & -153 \\
\hline & 0.18 & 0.03 & 0.77 & 0.79 & 9 \\
\hline & 0.30 & 0.28 & 1.14 & 1.22 & 43 \\
\hline & 0.19 & 0.02 & 1.21 & 1.22 & 6 \\
\hline & -0.04 & -0.24 & 1.23 & 1.26 & -99 \\
\hline & 0.40 & -0.60 & 1.07 & 1.29 & -56 \\
\hline \multirow[t]{7}{*}{ CN tor 1} & 0.22 & -0.13 & -3.47 & 3.48 & -31 \\
\hline & -0.50 & 0.43 & -3.39 & 3.45 & 139 \\
\hline & 0.37 & -0.46 & -3.37 & 3.42 & -51 \\
\hline & -0.19 & -0.27 & -2.36 & 2.38 & -125 \\
\hline & -0.11 & 0.04 & -2.22 & 2.23 & 160 \\
\hline & -0.03 & -0.04 & -2.43 & 2.43 & -128 \\
\hline & 0.07 & 0.68 & -2.02 & 2.13 & 84 \\
\hline \multirow[t]{7}{*}{$\mathrm{NC}^{\alpha}$ tor } & 0.17 & -0.07 & -3.36 & 3.36 & -24 \\
\hline & -0.19 & 0.81 & -4.90 & 4.97 & 103 \\
\hline & 0.13 & -1.03 & -5.19 & 5.29 & -83 \\
\hline & -0.96 & 0.20 & -3.86 & 3.98 & 168 \\
\hline & -0.55 & 0.16 & -3.31 & 3.36 & 164 \\
\hline & 0.80 & -0.08 & -3.40 & 3.50 & -6 \\
\hline & 1.40 & -0.20 & -3.07 & 3.38 & -8 \\
\hline
\end{tabular}


TABLE 6 (continued)

\begin{tabular}{|c|c|c|c|c|c|}
\hline & $\partial \mu_{x} / \partial S$ & $\partial \mu_{y} / \partial S$ & $\partial \mu_{2} / \partial S$ & $|\partial \vec{\mu} / \partial S|$ & $\theta^{\mathrm{b}}$ \\
\hline \multicolumn{6}{|c|}{ C12 N17 013} \\
\hline \multirow[t]{7}{*}{$\mathrm{NH}$ str2 } & 0.62 & 0.72 & -0.02 & 0.95 & 50 \\
\hline & 0.19 & 1.40 & -0.37 & 1.46 & 82 \\
\hline & 0.14 & 1.85 & 0.47 & 1.91 & 86 \\
\hline & 0.24 & 0.63 & 0.10 & 0.68 & 70 \\
\hline & 0.13 & 0.62 & 0.32 & 0.71 & 78 \\
\hline & 0.16 & 0.33 & -0.27 & 0.46 & 64 \\
\hline & 0.51 & 0.61 & -0.18 & 0.81 & 50 \\
\hline \multirow[t]{7}{*}{ COstr2 } & 4.19 & 4.79 & 0.23 & 6.37 & 49 \\
\hline & 4.47 & 4.68 & -0.37 & 6.48 & 46 \\
\hline & 4.45 & 4.96 & 0.12 & 6.67 & 48 \\
\hline & 4.24 & 4.82 & -0.10 & 6.42 & 49 \\
\hline & 4.33 & 4.95 & 0.05 & 6.58 & 49 \\
\hline & 4.24 & 4.84 & -0.37 & 6.44 & 49 \\
\hline & 3.87 & 4.95 & 0.12 & 6.29 & 52 \\
\hline \multirow[t]{7}{*}{$\mathrm{CN}$ str2 } & -4.42 & -0.40 & 0.09 & 4.44 & -175 \\
\hline & -3.87 & -0.30 & 0.21 & 3.89 & -176 \\
\hline & -3.52 & -0.43 & -0.28 & 3.56 & -173 \\
\hline & -4.18 & -0.74 & -0.01 & 4.24 & -170 \\
\hline & -3.91 & -0.96 & -0.25 & 4.04 & -166 \\
\hline & -3.88 & -0.80 & 0.16 & 3.96 & -168 \\
\hline & -3.83 & -1.10 & -0.02 & 3.99 & -164 \\
\hline \multirow[t]{7}{*}{$\mathrm{C}^{\alpha} \mathrm{C}$ str } & 0.30 & 0.16 & -0.19 & 0.39 & 28 \\
\hline & 0.62 & -0.53 & 0.43 & 0.92 & -41 \\
\hline & 0.97 & -0.87 & -0.22 & 1.32 & -42 \\
\hline & 0.41 & -0.32 & -0.00 & 0.52 & -38 \\
\hline & 0.60 & -0.42 & 0.19 & 0.75 & -35 \\
\hline & 0.24 & -0.14 & -0.48 & 0.55 & -30 \\
\hline & 0.52 & -0.37 & -0.02 & 0.64 & -35 \\
\hline \multirow[t]{7}{*}{$\mathrm{C}^{\alpha} \mathrm{CN}$ def } & 1.08 & -1.42 & -0.02 & 1.78 & -53 \\
\hline & 0.40 & -2.96 & 0.34 & 3.01 & -82 \\
\hline & 0.20 & -3.07 & -0.31 & 3.09 & -86 \\
\hline & 0.54 & -1.68 & 0.06 & 1.76 & -72 \\
\hline & -0.47 & -1.35 & -0.17 & 1.44 & -109 \\
\hline & -1.06 & -0.80 & -0.11 & 1.33 & -143 \\
\hline & 0.55 & -0.11 & -0.52 & 0.77 & -11 \\
\hline \multirow[t]{7}{*}{$\mathrm{CO}$ ib2 } & -3.23 & 2.41 & 0.05 & 4.03 & 143 \\
\hline & -2.58 & 2.40 & -0.05 & 3.53 & 137 \\
\hline & -2.21 & 2.30 & -0.11 & 3.19 & 134 \\
\hline & -2.68 & 2.28 & -0.11 & 3.52 & 140 \\
\hline & -2.37 & 2.17 & -0.14 & 3.22 & 137 \\
\hline & -2.41 & 2.16 & -0.11 & 3.24 & 138 \\
\hline & -2.57 & 1.82 & 0.20 & 3.16 & 145 \\
\hline
\end{tabular}


TABLE 6 (continued)

\begin{tabular}{|c|c|c|c|c|c|}
\hline & $\partial \mu_{x} / \partial S$ & $\partial \mu_{y} / \partial S$ & $\partial \mu_{z} / \partial S$ & $|\partial \vec{\mu} / \partial S|$ & $\theta^{\mathbf{b}}$ \\
\hline \multirow[t]{7}{*}{ CNM def } & 0.73 & 0.32 & 0.01 & 0.79 & 24 \\
\hline & 0.99 & 0.84 & -0.06 & 1.30 & 40 \\
\hline & 1.15 & 0.87 & 0.02 & 1.44 & 37 \\
\hline & 0.88 & 0.42 & -0.04 & 0.97 & 26 \\
\hline & 1.36 & 0.27 & -0.01 & 1.39 & 11 \\
\hline & 1.47 & 0.12 & 0.07 & 1.47 & 5 \\
\hline & 0.91 & -0.05 & 0.29 & 0.96 & -3 \\
\hline \multirow[t]{7}{*}{ NH ib2 } & 0.59 & -0.12 & -0.03 & 0.61 & -12 \\
\hline & 0.73 & -0.45 & 0.27 & 0.90 & -32 \\
\hline & 0.66 & -0.54 & -0.18 & 0.87 & -39 \\
\hline & 0.59 & -0.41 & 0.04 & 0.72 & -35 \\
\hline & 0.55 & -0.46 & -0.07 & 0.72 & -40 \\
\hline & 0.57 & -0.27 & 0.13 & 0.65 & -25 \\
\hline & 0.59 & -0.24 & -0.05 & 0.64 & -22 \\
\hline \multirow[t]{7}{*}{$\mathrm{CO}$ ob2 } & -0.05 & -0.02 & 0.85 & 0.85 & -160 \\
\hline & 0.06 & -0.21 & -0.33 & 0.39 & -75 \\
\hline & -0.08 & 0.17 & -0.38 & 0.43 & 115 \\
\hline & 0.72 & -0.56 & 0.79 & 1.21 & -38 \\
\hline & 0.94 & -0.98 & 0.28 & 1.39 & -46 \\
\hline & -0.85 & 0.46 & -0.07 & 0.97 & 151 \\
\hline & -0.40 & 0.25 & 1.10 & 1.20 & 148 \\
\hline \multirow[t]{7}{*}{ NH ob2 } & -0.00 & 0.09 & 2.24 & 2.24 & 91 \\
\hline & 0.20 & 0.08 & 2.66 & 2.67 & 22 \\
\hline & -0.05 & -0.05 & 2.70 & 2.70 & -139 \\
\hline & -0.32 & 0.09 & 2.07 & 2.10 & 164 \\
\hline & -0.68 & 0.22 & 2.22 & 2.33 & 162 \\
\hline & 0.73 & -0.25 & 2.39 & 2.51 & -19 \\
\hline & 0.45 & 0.28 & 1.90 & 1.98 & 31 \\
\hline \multirow[t]{7}{*}{$\mathrm{C}^{\alpha} \mathrm{C}$ tor } & 0.11 & -0.15 & 3.38 & 3.39 & -52 \\
\hline & -0.01 & -0.29 & 4.37 & 4.38 & -91 \\
\hline & 0.11 & 0.51 & 4.32 & 4.35 & 78 \\
\hline & -0.74 & -0.30 & 2.74 & 2.85 & -158 \\
\hline & -1.19 & 0.30 & 3.24 & 3.47 & 166 \\
\hline & 1.23 & -0.83 & 2.90 & 3.26 & -34 \\
\hline & 0.42 & 0.50 & 1.76 & 1.88 & 50 \\
\hline \multirow[t]{7}{*}{$\mathrm{CN}$ tor2 } & -0.11 & 0.08 & -0.06 & 0.15 & 143 \\
\hline & 0.34 & -0.38 & -0.33 & 0.61 & -48 \\
\hline & -0.19 & 0.36 & -0.41 & 0.58 & 118 \\
\hline & 0.51 & -0.47 & -0.01 & 0.69 & -42 \\
\hline & 0.54 & -0.52 & -0.43 & 0.86 & -44 \\
\hline & -0.29 & 0.36 & -0.41 & 0.62 & 128 \\
\hline & -0.08 & 0.27 & 0.30 & 0.41 & 108 \\
\hline
\end{tabular}


TABLE 6 (continued)

\begin{tabular}{|c|c|c|c|c|c|}
\hline & $\partial \mu_{x} / \partial S$ & $\partial \mu_{y} / \partial S$ & $\partial \mu_{z} / \partial S$ & $|\partial \vec{\mu} / \partial S|$ & $\theta^{\mathbf{b}}$ \\
\hline \multicolumn{6}{|c|}{$\mathrm{C} 11 \mathrm{C} 9 \mathrm{H} 16$} \\
\hline \multirow[t]{7}{*}{$\mathrm{C}^{\alpha} \mathrm{C}^{\beta}$ str } & 0.17 & 0.47 & 0.46 & 0.68 & 71 \\
\hline & 0.23 & 0.19 & 0.68 & 0.75 & 40 \\
\hline & 0.24 & 0.33 & 0.56 & 0.69 & 55 \\
\hline & 0.29 & 0.39 & 0.56 & 0.74 & 54 \\
\hline & 0.40 & 0.67 & 0.65 & 1.02 & 59 \\
\hline & 0.26 & -0.08 & 0.77 & 0.82 & -18 \\
\hline & 0.41 & 0.59 & 0.45 & 0.85 & 55 \\
\hline \multirow[t]{7}{*}{$\mathrm{C}^{\alpha} \mathrm{H}^{\alpha}$ str } & -0.05 & 0.36 & 0.04 & 0.36 & 98 \\
\hline & -0.13 & 0.08 & -0.35 & 0.39 & 150 \\
\hline & -0.00 & -0.08 & -0.15 & 0.17 & -93 \\
\hline & -0.19 & 0.39 & -0.46 & 0.63 & 115 \\
\hline & -0.02 & 0.07 & -0.37 & 0.38 & 101 \\
\hline & -0.31 & 0.08 & 0.07 & 0.32 & 166 \\
\hline & 0.03 & 0.13 & -0.15 & 0.20 & 79 \\
\hline \multirow[t]{7}{*}{$\mathrm{NC}^{\alpha} \mathrm{C}$ def } & -2.03 & 1.95 & -0.27 & 2.82 & 136 \\
\hline & -2.29 & 1.93 & -0.14 & 3.00 & 140 \\
\hline & 3.84 & -2.08 & -0.29 & 4.37 & -29 \\
\hline & -1.92 & 1.06 & 0.65 & 2.28 & 151 \\
\hline & -0.40 & 0.21 & 0.21 & 0.50 & 152 \\
\hline & 0.54 & -0.08 & -0.10 & 0.55 & -9 \\
\hline & -0.24 & -0.23 & 0.79 & 0.86 & -136 \\
\hline \multirow[t]{7}{*}{$\mathrm{H}^{\alpha} \mathrm{b} \mathbf{1}$} & -0.02 & -0.03 & 0.04 & 0.05 & -126 \\
\hline & -1.23 & 0.65 & 0.12 & 1.39 & 152 \\
\hline & 1.03 & -1.11 & 0.10 & 1.51 & -47 \\
\hline & -0.66 & 0.40 & 0.87 & 1.16 & 149 \\
\hline & -0.27 & 0.41 & 0.55 & 0.74 & 124 \\
\hline & -0.27 & -0.67 & 0.26 & 0.77 & -112 \\
\hline & 0.69 & -0.10 & 0.91 & 1.15 & -8 \\
\hline \multirow[t]{7}{*}{$\mathrm{H}^{\alpha} \mathrm{b} 2$} & 0.15 & -0.05 & 1.09 & 1.10 & -19 \\
\hline & 0.61 & 0.85 & -0.23 & 1.07 & 55 \\
\hline & -0.51 & -0.97 & -0.60 & 1.25 & -118 \\
\hline & 0.68 & 0.39 & 0.12 & 0.79 & 30 \\
\hline & 0.09 & 0.51 & -0.50 & 0.72 & 80 \\
\hline & 0.21 & -0.52 & -0.84 & 1.01 & -68 \\
\hline & -0.03 & -0.15 & 0.36 & 0.39 & -101 \\
\hline \multirow[t]{7}{*}{$\mathrm{C}^{\beta} \mathrm{b} 1$} & -3.26 & 2.20 & -0.14 & 3.94 & 146 \\
\hline & 1.56 & -1.90 & -0.46 & 2.50 & -51 \\
\hline & -0.02 & -0.98 & 0.18 & 0.99 & -92 \\
\hline & -0.22 & -1.80 & -0.17 & 1.82 & -97 \\
\hline & 0.82 & -1.47 & -0.34 & 1.72 & -61 \\
\hline & 0.49 & -1.23 & 0.11 & 1.33 & -68 \\
\hline & -2.35 & 0.08 & -0.29 & 2.37 & 178 \\
\hline
\end{tabular}


TABLE 6 (continued)

\begin{tabular}{rrrrrr}
\hline & $\partial \mu_{x} / \partial S$ & $\partial \mu_{y} / \partial S$ & $\partial \mu_{z} / \partial S$ & $|\partial \vec{\mu} / \partial S|$ & \multicolumn{1}{c}{$\theta^{\mathbf{b}}$} \\
\hline $\mathrm{C}^{\beta} \mathrm{b} 2$ & 0.20 & -0.32 & -0.58 & 0.69 & -57 \\
& -0.48 & -1.46 & 0.31 & 1.56 & -108 \\
& 0.61 & 0.65 & 0.32 & 0.95 & 47 \\
& 0.00 & -1.12 & -0.23 & 1.15 & -90 \\
& -0.11 & -1.14 & 0.16 & 1.16 & -96 \\
& -0.11 & -0.45 & 0.63 & 0.78 & -104 \\
& 0.55 & -0.31 & -0.32 & 0.70 & -29
\end{tabular}

${ }^{a}$ Local axes of group $\mathrm{A} \mathrm{B} \mathrm{C}$ defined thus: $\hat{x}=\mathrm{AB}, \hat{z}=\mathrm{AC} \times \mathrm{AB}$. Entries are for $\mathrm{C}_{5}$ (first line), $\mathrm{C}_{7}^{\text {eq }}$, $\mathrm{C}_{7}^{\mathrm{ax}}, \beta_{2}, \alpha_{\mathrm{R}}, \alpha_{\mathrm{L}}$, and $\alpha^{\prime}$ (subsequent lines).

${ }^{\mathrm{b}}$ Angle from $\hat{x}$ in $x-y$ plane, in degrees.

found in our experimental study of cyclo (D-Ala-L-Ala) in two crystalline forms [19]. The precise nature of these frequency-conformation correlations is still not clear, given the small number of data points available at present. However, the $\mathrm{CH}^{\alpha}$ stretch frequency does seem to decrease with $\psi$, which is consistent with our experimental observation [19] of a $21 \mathrm{~cm}^{-1}$ decrease when $\psi$ changes from $-2.8^{\circ}$ to $4.2^{\circ}$ in a cis peptide.

The sets of frequencies for all seven conformers of Ala dipeptide yield the following vibrational free energies at $298.15 \mathrm{~K}$ (using the usual harmonic oscillator expression [20], in kcal mol-1): $109.68\left(\mathrm{C}_{5}\right), 109.85\left(\mathrm{C}_{7}^{\text {eq }}\right), 110.30$ $\left(\mathrm{C}_{7}^{\mathrm{ax}}\right), 109.55\left(\beta_{2}\right), 109.45\left(\alpha_{\mathrm{R}}\right), 109.66\left(\alpha_{\mathrm{L}}\right)$, and $109.51\left(\alpha^{\prime}\right)$. The free energies, given by the sum of the SCF energy and the vibrational free energy, in units of kcal mol ${ }^{-1}$ relative to that of $\mathrm{C}_{7}^{\mathrm{eq}}$, are: $1.2\left(\mathrm{C}_{5}\right), 3.1\left(\mathrm{C}_{7}^{\mathrm{ax}}\right), 3.6\left(\beta_{2}\right), 4.5$ $\left(\alpha_{\mathrm{R}}\right), 6.5\left(\alpha_{\mathrm{L}}\right)$, and 7.6 $\left(\alpha^{\prime}\right)$. Thus, the order of stability of the conformers is unchanged from that given by the SCF energies alone, and each conformer is slightly closer in energy to the $\mathrm{C}_{7}^{\text {eq }}$ except for the $\mathrm{C}_{7}^{\text {ax }}$ structure.

Table 6 lists the dipole-moment derivatives $\partial \mu / \partial S_{i}$ with respect to the group coordinates (excluding those of the methyl groups) referred to local axes fixed within each group [1]; see the footnote to the table for the definitions of the local axes. To help in comparison, all seven Ala dipeptide conformers are tabulated.

The NH strl derivatives show marked variations in the open conformers and, unlike in the $\mathrm{C}_{5}$ and $\mathrm{C}_{7}$ structures, have sizeable components perpendicular to the plane of the peptide group; this is probably related to the deviations from planarity at $\mathrm{N} 7$ (Table 1). The NH str2 derivatives clearly show a strong increase due to the $\mathrm{C}_{7}$ hydrogen bonds. Except for CO str1 in $\alpha_{\mathrm{L}}$, the CO stretch derivatives are all very similar in magnitude and direction even in the hydrogen-bonded structures, pointing to a high degree of transferability. (Because $\mathrm{CO}$ str2 is more representative of the $\mathrm{CO}$ groups in polypeptides, the $\alpha_{\mathrm{L}}$ exception seems of little consequence.) 
The $\mathrm{NH}$ ib derivatives are not very conformationally sensitive, at least in their magnitudes, and as expected they increase when the $\mathrm{NH}$ group is bonded. All $\mathrm{CO} \mathrm{ib} 1$ and $\mathrm{CO}$ ib2 derivatives have nearly the same magnitude and direction. The $\mathrm{NH}$ ob1 derivatives are systematically smaller in magnitude than the $\mathrm{NH}$ ob2 and in the open conformers the former are all virtually identical. (Note that $\mathrm{NH}$ ob1 is the more representative of $\mathrm{NH}$ groups in polypeptides.) There is the expected increase on hydrogen bonding and interestingly, a decrease in $\mathrm{NH}$ ob1 in the $\mathrm{C}_{7}$ structures where the $\mathrm{NH}$ belongs to the acceptor peptide unit. Similar decreases in the $\mathrm{CO}$ ob derivatives are seen when the $\mathrm{CO}$ is in the donor peptide group. The situation in proteins and peptide crystals, where each $\mathrm{CONH}$ is usually both a hydrogen-bond donor and acceptor, is likely to be more complicated.

\section{CONCLUSIONS}

We have extended our previous studies of the force fields of Ala dipeptide in $\mathrm{C}_{5}$ and $\mathrm{C}_{7}$ conformations with intramolecular hydrogen bonding by doing similar calculations on four open, non-hydrogen-bonded conformations. These results have provided further insights into how force constants vary with conformation and hydrogen bonding, and should be helpful in developing and testing force fields for normal mode and molecular mechanics calculations of peptides and polypeptides.

\section{ACKNOWLEDGEMENT}

This research was supported by NSF grants DMB-8816756 and DMR8806975. We thank The University of Michigan Computing Center for a generous special allocation of computing funds.

\section{REFERENCES}

1 T.C. Cheam and S. Krimm, J. Mol. Struct., 193 (1989) 1. (In Table 3 of this reference, the 2-33 element for GLY7 should read -0.03 , and in Table 8 the last group heading should read C16N14H19.)

2 T.C. Cheam and S. Krimm, J. Mol. Struct. (Theochem), 188 (1989) 15. (In Table 3 of this reference, the 4-30 and 4-23 terms should be interchanged, and the 17-41 and 17-42 elements for ALA 7A should read 0.05 and 0.10 , respectively.)

3 L. Schäfer, C. Van Alsenoy and J.N. Scarsdale, J. Chem. Phys., 76 (1982) 1439.

4 J.N. Scarsdale, C. Van Alsenoy, V.J. Klimkowski, L. Schäfer and F.A. Momany, J. Am. Chem. Soc., 105 (1983) 3438.

5 L. Schäfer, V.J. Klimkowski, F.A. Momany, H. Chuman and C. Van Alsenoy, Biopolymers, 23 (1984) 2335.

6 V.J. Klimkowski, L. Schäfer, F.A. Momany and C: Van Alsenoy, J. Mol. Struct. (Theochem), 124 (1985) 143. 
7 K. Rasmussen, Potential Energy Functions in Conformational Analysis, Springer-Verlag, Rerlin, 1985.

8 P. Pulay, G. Fogarasi, F. Pang and J.E. Boggs, J. Am. Chem. Soc., 101 (1979) 2550.

9 E.B. Wilson, Jr., J.C. Decius and P.C. Cross, Molecular Vibrations, Dover, New York, 1980, p. 60 .

10 G. Fogarasi and A. Balázs, J. Mol. Struct. (Theochem), 133 (1985) 105.

11 M. Marraud, J. Néel, M. Avignon and P.V. Huong, J. Chim. Phys., 67 (1970) 959.

12 J. Néel, Pure Appl. Chem., 31 (1972) 201.

13 I.M. Ginzburg, J. Gen. Chem., 52 (1982) 1445.

14 R.P. Sheridan, R.H. Lee, N. Peters and L.C. Allen, Biopolymers, 18 (1979) 2451.

15 T. Ashida, Y. Tsunogae, I. Tanaka and T. Yamane, Acta Crystallogr., Sect. B, 43 (1987) 212.

16 Y. Harada and Y. Iitaka, Acta Crystallogr., Sect. B., 30 (1974) 1452.

17 S. Krimm and J. Bandekar, Adv. Protein Chem., 38 (1986) 181.

18 T.C. Cheam and S. Krimm, Chem. Phys. Lett., 107 (1984) 613.

19 T.C. Cheam and S. Krimm, Spectrochim. Acta, Part A, 44 (1988) 185.

20 J.C. Slater, Introduction to Chemical Physics, McGraw-Hill, New York, 1939, p. 217. 